\title{
THE FIRST INFRARED STUDY OF THE CLOSE ENVIRONMENT OF A LONG GAMMA-RAY BURST*
}

\author{
Emeric Le Floc' ${ }^{1}{ }^{1}$, Vassilis Charmandaris ${ }^{2,3,4}$, Karl Gordon ${ }^{5}$, William J. Forrest ${ }^{6}$, Bernhard Brandl $^{7}$, \\ Daniel Schaerer $^{8,9}$, Miroslava Dessauges-Zavadsky $^{8}$, and LeE Armus $^{10}$ \\ ${ }^{1}$ Laboratoire AIM, CEA/DSM/IRFU, CNRS, Université Paris-Diderot, 91190 Gif, France \\ ${ }^{2}$ Department of Physics and Institute of Theoretical and Computational Physics, University of Crete, GR-71002, Heraklion, Greece \\ ${ }^{3}$ Chercheur Associé, Observatoire de Paris, F-75014 Paris, France \\ ${ }^{4}$ IESL/Foundation for Research and Technology-Hellas, P.O. Box 1527, 71110 Heraklion, Greece \\ ${ }^{5}$ Space Telescope Science Institute, 3700 San Martin Drive, Baltimore, MD 21218, USA \\ ${ }^{6}$ Department of Physics and Astronomy, University of Rochester, Rochester, NY 14627, USA \\ ${ }^{7}$ Leiden Observatory, Leiden University, Niels Bohrweg 2, P.O. Box 9513, 2300 RA Leiden, The Netherlands \\ ${ }^{8}$ Geneva Observatory, University of Geneva, 51, Ch. des Maillettes, CH-1290 Versoix, Switzerland \\ ${ }^{9}$ CNRS, IRAP, 9 Av. Colonel Roche, BP 44346, F-31028 Toulouse, Cedex 4, France \\ ${ }^{10}$ Spitzer Science Center, California Institute of Technology, 220-6 Pasadena, CA 91125, USA \\ Received 2011 August 24; accepted 2011 November 3; published 2012 January 18
}

\begin{abstract}
We present a characterization of the close environment of GRB 980425 based on 5-160 $\mu$ m spectro-imaging obtained with Spitzer. The gamma-ray burst GRB 980425 occurred in a nearby $(z=0.0085)$ SBc-type dwarf galaxy at a projected distance of 900 pc from an H II region with strong signatures of Wolf-Rayet (WR) stars. While this "WR region" produces less than $5 \%$ of the $B$-band emission of the host, we find that it is responsible for $45 \% \pm 10 \%$ of the total infrared luminosity, with a maximum contribution reaching $75 \%$ at $25-30 \mu \mathrm{m}$. This atypical property is rarely observed among morphologically relaxed dwarfs, suggesting a strong causal link with the gamma-ray burst (GRB) event. The luminosity of the WR region $\left(L_{8-1000 \mu \mathrm{m}}=4.6 \times 10^{8} L_{\odot}\right)$, the peak of its spectral energy distribution at $\lesssim 100 \mu \mathrm{m}$, and the presence of highly ionized emission lines (e.g., [Ne III]) also reveal extremely young ( $<5$ Myr) star-forming activity, with a typical timescale of only 47 Myr to double the stellar mass already built. Finally, the mid-IR over $B$-band luminosity ratio in this region is substantially higher than in star-forming galaxies with similar $L_{\mathrm{IR}}$, but it is lower than in young dust-enshrouded stellar clusters. Considering the modest obscuration measured from the silicate features $\left(\tau_{9.7 \mu \mathrm{m}} \sim 0.015\right)$, this suggests that the WR region is dominated by one or several star clusters that have either partly escaped or cleared out their parent molecular cloud. Combined with the properties characterizing the whole population of GRB hosts, our results reinforce the idea that long GRBs mostly happen within or in the vicinity of relatively unobscured galactic regions harboring very recent star formation.
\end{abstract}

Key words: galaxies: individual (GRB980425) - infrared: galaxies

Online-only material: color figures

\section{INTRODUCTION}

It is now widely believed that long gamma-ray bursts $\left(\mathrm{LGRBs}^{11}\right.$ ) originate from the cataclysmic collapse of massive stars (e.g., Woosley 1993; Galama et al. 1998; MacFadyen \& Woosley 1999; Daigne \& Mochkovitch 2000; Heger et al. 2003; Stanek et al. 2003; Meynet \& Maeder 2005) in star-forming regions of distant galaxies (e.g., Fruchter 1999; Bloom et al. 2002; Hjorth et al. 2002; Fruchter et al. 2006). They can be observed at extremely high redshift ( $z>6$; Greiner et al. 2009) and because of their association with young and short-lived stars, they are invaluable tools for sign-posting the activity of star formation back to the earliest epochs of cosmic history and galaxy evolution. Furthermore, their optical and near-infrared afterglows can be used as transient background sources for probing the intergalactic space and the interstellar medium (ISM) of galaxies up to very large cosmological distances (e.g., Vreeswijk et al. 2001; Totani et al. 2006). As a result, they might eventually provide unique constraints on the era of reionization and the

\footnotetext{
* Based on observations made with Spitzer, operated by the Jet Propulsion Laboratory under NASA contract 1407.

11 Long GRBs, referred as LGRBs in this paper, are defined as gamma-ray bursts with a duration greater than $\sim 2 \mathrm{~s}$ and a soft spectrum, as opposed to the class of the short and hard GRBs (Kouveliotou et al. 1993).
}

formation of the very first structures in the universe (Lamb \& Reichart 2000).

However, it appears that LGRBs may only trace regions of star-forming activity in a biased way, in the sense that their formation is more likely to occur in low-mass and sub$L^{*}$ galaxies with low-metallicity environments (Sokolov et al. 2001; Fynbo et al. 2003; Courty et al. 2004, 2007; Kistler et al. 2009; Kocevski et al. 2009; Han et al. 2010; Niino et al. 2011). In the latest stages of their evolution, massive stars with metalpoor envelopes retain a higher angular momentum and they are less subject to mass loss. After the final collapse, these physical conditions lead to the formation of fast-rotating black holes and the subsequent accretion of material thus favors the formation of GRBs (e.g., MacFadyen \& Woosley 1999; Hirschi et al. 2005). In fact, observational evidence for a possible relationship between LGRBs and chemically young galaxies has already emerged from a number of studies. On average the hosts of LGRBs are characterized by blue colors, rather low extinction and modest bolometric luminosities, as well as diffuse and irregular morphologies (e.g., Bloom et al. 2002; Le Floc'h et al. 2003, 2006; Fruchter et al. 2006). They also exhibit higher Ly $\alpha$ emission and larger specific star formation rates (SSFRs) with respect to other galaxies at similar redshifts (Fynbo et al. 2003; Christensen et al. 2004), and their oxygen abundance is lower compared to field star-forming sources 
with similar masses and luminosities (Modjaz et al. 2008; Han et al. 2010). Furthermore, the connection between GRBs and Type Ic supernovae has led to the idea that the progenitors of LGRBs should more likely originate from binary systems (Podsiadlowski et al. 2004), which complicate further the link that exists between LGRBs and the cosmic history of structure formation. Better understanding the physical conditions and the properties of the environments favoring the formation of these cosmic explosions appears, therefore, as a critical step before allowing a full exploitation of their hosts as cosmological tracers of galaxy evolution.

The follow-up of gamma-ray bursts (GRBs) has shown that high-redshift LGRB hosts are challenging to characterize in detail, since they tend to be low-mass and sub-luminous systems (e.g., Savaglio et al. 2009). Host galaxies at very low redshift may thus provide us with some of the best cases to explore the intimate connection between the occurrence of GRBs and the properties of their very close environment. For example, the now-popular GRB 980425 was identified at $z=0.0085$ from its apparent association with the hypernova SN 1998bw (Galama et al. 1998). With a luminosity distance of only $36 \mathrm{Mpc}$, it is currently the closest GRB known with a confirmed redshift in the local universe. At this distance, an angular separation of $1^{\prime \prime}$ on the sky corresponds to $170 \mathrm{pc}$. It provides therefore an excellent prototype for studying the immediate surrounding of LGRBs within their host galaxies.

The host of GRB 980425 is identified as ESO 184-G82 in the survey of the southern sky that was performed by Holmberg et al. (1977). Observations with the Hubble Space Telescope (HST) showed that it is an isolated and barred SBc-type subluminous galaxy $\left(L_{\mathrm{B}}=0.02 L_{\mathrm{B}}^{*}\right)$ harboring a large number of active star-forming regions (Fynbo et al. 2000). While the surface brightness of the area where GRB 980425 was observed is not particularly large, a remarkable result is that the GRB occurred at a projected distance of only $\sim 900 \mathrm{pc}$ from a quite luminous $\mathrm{H}$ II region characterized by very distinct properties compared to the rest of the system. This region is the brightest complex of star formation in the host and although it only makes a few percent of the total luminosity at optical wavelengths, infrared (IR) imaging obtained with the Spitzer Space Telescope revealed that its 5-25 $\mu \mathrm{m}$ spectral energy distribution (SED) is characterized by a steeply rising contribution with respect to the whole SED of the galaxy (Le Floc'h et al. 2006). At $24 \mu \mathrm{m}$, it is responsible for a fraction as large as $\sim 75 \%$ of the monochromatic luminosity of the entire system. Among low-luminosity star-forming disks and morphologically evolved dwarf galaxies, such a high contribution of extranuclear star formation is rarely observed. It makes the host of GRB 980425 an atypical object of the local universe.

While the stellar mass already assembled in this region is low ( $~ 6 \times 10^{6} M_{\odot}$, Michałowski et al. 2009), long-slit spectroscopy revealed the presence of numerous Wolf-Rayet (WR) stars as well as prominent $\mathrm{H} \alpha$ and $\mathrm{H} \beta$ emission lines with very large equivalent widths (EWs; Hammer et al. 2006). A metallicity of $Z \sim 0.5 Z_{\odot}$ was inferred from the effective temperature of the ionized gas (Hammer et al. 2006) and the estimated star formation rates (SFRs) vary between 0.06 and $0.3 M_{\odot} \mathrm{yr}^{-1}$ (Le Floc'h et al. 2006; Hammer et al. 2006; Christensen et al. 2008; Michałowski et al. 2009). This implies a large SSFR and suggests that this environment (denoted as "WR region" hereafter) is experiencing a young and vigorous starburst. Finally, integral field spectroscopy performed by Christensen et al. (2008) with the VIsible Multi Object Spectrograph
(VIMOS) on the Very Large Telescope revealed that the ionizing conditions in this area are markedly different from those observed in the other $\mathrm{HII}$ regions found in the host. With a particularly strong $[\mathrm{O} \mathrm{III}] / \mathrm{H} \beta$ line ratio $(\gtrsim 5)$, the ionized gas properties observed in the WR region are typical of very young starbursts at sub-solar metallicities, and they also appear very similar to the properties characterizing the more distant GRB host galaxies as a whole. Along with the detection of WR stars considered as very strong candidates of collapsar progenitors, the emission line diagnostics suggest therefore an intimate connection between the characteristics of this source and the physical mechanisms triggering the formation of LGRBs in the universe. Hammer et al. (2006) even proposed that the WR region may have been the true birthplace of the GRB 980425 progenitor. The latter would have been dynamically ejected from this environment to finally produce a collapsar after traveling at velocities of $\sim 200-300 \mathrm{~km} \mathrm{~s}^{-1}$ during a few Myr through the ISM of the galaxy.

Following our previous characterization of the GRB 980425 host based on $4.5 \mu \mathrm{m}, 8 \mu \mathrm{m}$, and $24 \mu \mathrm{m}$ broadband imaging (Le Floc'h et al. 2006), we performed additional IR follow-up with the Spitzer Space Telescope in order to further constrain the spectroscopic mid-IR properties as well as the far-IR luminosity of the WR region and the host galaxy. Here we report on the $5-35 \mu \mathrm{m}$ spectrum that we obtained with the Infrared Spectrograph (IRS) for Spitzer (Houck et al. 2004b) as well as the $70 \mu \mathrm{m}$ and $160 \mu \mathrm{m}$ imaging obtained with the Multiband Imager and Photometer (MIPS) for Spitzer (Rieke et al. 2004). Along with the host of GRB $031203(z=0.1055)$ that was also observed with the IRS by Watson et al. (2011), ESO 184-G82 represents one of the very few identifications of LGRB host galaxies located close enough to allow detailed characterization in the thermal infrared. Our observations are described in Section 2, while in Section 3 we present the broadband photometry and the spectroscopic measurements derived from the Spitzer data. In Section 4 we derive the total IR SEDs and SFRs of the host and the WR region, and in Section 5 we constrain in more detail their mid-IR spectroscopic properties. We discuss our results in Section 6 and we present our conclusions in Section 7. Throughout this work, physical quantities are computed assuming $H_{0}=70 \mathrm{~km} \mathrm{~s}^{-1}$ as well as the initial mass function (IMF) from Salpeter (1955).

\section{OBSERVATIONS AND DATA REDUCTION}

\subsection{Far-infrared Imaging}

The host galaxy of GRB 980425 was imaged at $70 \mu \mathrm{m}$ and $160 \mu \mathrm{m}$ with the MIPS instrument as part of a Spitzer Cycle 3 General Observer program (PID: 30 251, AOR keys 24966656 and 24966400). The observations were carried out in 2008 using the MIPS "Small Scale Photometry" mode. With this operating mode, a set of 10 frames is taken for each observing cycle and a dither is automatically performed around the targeted source. We respectively obtained 5 and 12 cycles at $70 \mu \mathrm{m}$ and $160 \mu \mathrm{m}$, which resulted in a total on-source integration time of $545 \mathrm{~s}$ at $70 \mu \mathrm{m}$ and $250 \mathrm{~s}$ at $160 \mu \mathrm{m}$.

For a very modest amount of additional requested time we also performed a new observation of the GRB 980425 host at $24 \mu \mathrm{m}$, with the goal of checking if some fraction of the luminosity of the bright $24 \mu \mathrm{m}$ point source detected in our previous data (Le Floc'h et al. 2006) could be associated with the emission from a transient object (e.g., supernova). A single cycle of 10 frames was obtained, leading to a total integration time of $165 \mathrm{~s}$ on source. 


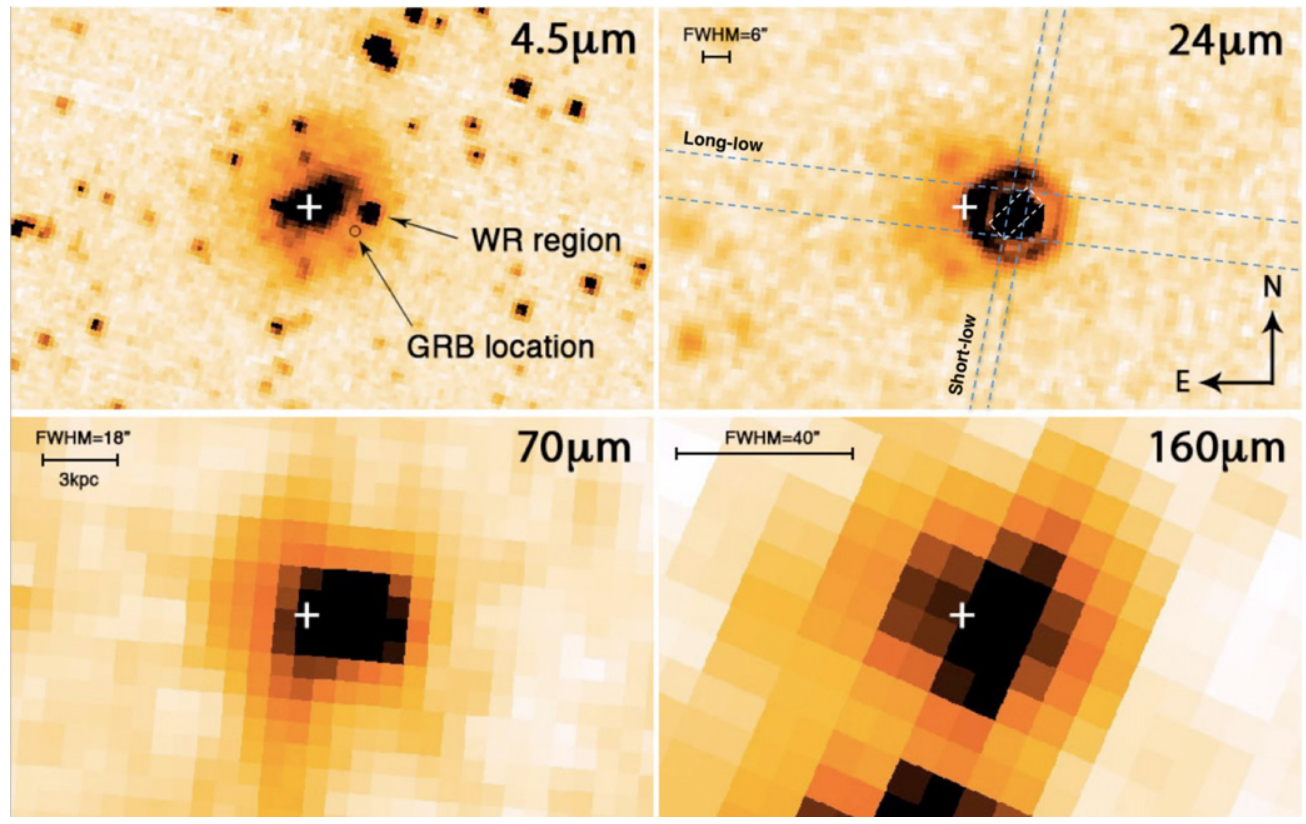

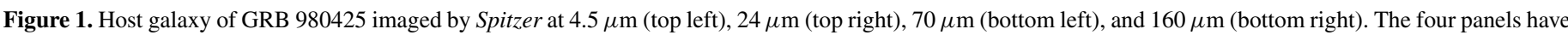

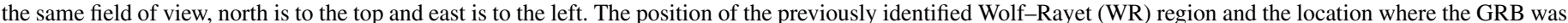

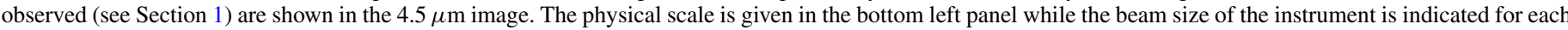

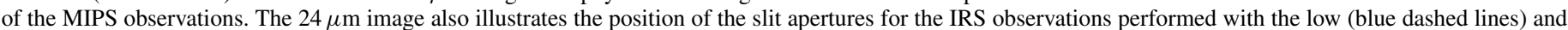

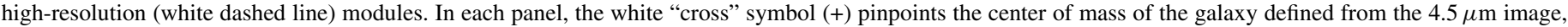
which shows that the strong emission detected at $70 \mu \mathrm{m}$ and $160 \mu \mathrm{m}$ is shifted toward the WR region.

(A color version of this figure is available in the online journal.)

Each individual image was processed with the most recent version (v.3.1) of the MIPS Data Analysis Tool (DAT; Gordon et al. 2005). This update includes additional processing steps with respect to the first version of the DAT initially released. In particular, a pixel-dependent background subtraction was applied to each individual frame before co-adding the images. We used calibration factors of $702\left(\mathrm{MJy} \mathrm{sr}^{-1}\right) /(\mathrm{ADU})$ and $41.7\left(\mathrm{MJy} \mathrm{sr}^{-1}\right) /(\mathrm{ADU})$ at $70 \mu \mathrm{m}$ and $160 \mu \mathrm{m}$, respectively (Gordon et al. 2007; Stansberry et al. 2007). The pixel size of the final mosaics was set to half the pixel size of the MIPS detectors and the absolute astrometric registration was tied to the Spitzer astrometry of the raw data as provided by the Spitzer Science Center. The images obtained at the three MIPS wavelengths are presented in Figure 1. For reference, they are compared with the image of the GRB 980425 host galaxy obtained at $4.5 \mu \mathrm{m}$ from our first Spitzer observations (Le Floc'h et al. 2006).

\subsection{Mid-infrared Spectroscopy}

The mid-IR spectroscopic follow-up of the WR region was performed as part of the IRS Guaranteed Time Observation program on 2006 April 19 (AOR key: 12246528). The target was first acquired using the red peak-up camera. During this acquisition an image of the source at $22 \mu \mathrm{m}$ was obtained in double correlated sampling mode to locate the mid-IR centroid of the target, and then offset to the appropriate slit (see the Spitzer Observers Manual for more details). Using the short-low (SL; 5.2-14.5 $\mu \mathrm{m}$ ) and long-low (LL; 14.0-38.0 $\mu \mathrm{m}$ ) modules of the IRS, we obtained a $5 \sim 38 \mu \mathrm{m}$ low-resolution spectrum of the WR region. The total on-source exposure time was $244 \mathrm{~s}$ ( 2 cycles of $60 \mathrm{~s}$ ) per SL order and $126 \mathrm{~s}$ ( 2 cycles of $30 \mathrm{~s}$ ) per LL order. In addition, higher resolution observations were obtained with the IRS short-high (SH; 9.9-19.6 $\mu \mathrm{m}$ ) for a total on-source time of $975 \mathrm{~s}$ ( 4 cycles of $120 \mathrm{~s}$ ). For each mode of our IRS observations, the orientation of the slit aperture with respect to the GRB 980425 host galaxy is illustrated in Figure 1 (top right panel).

We begun analyzing the observations by processing the data with the Spitzer Science Center pipeline (version 15.5) and the extraction of the signal was carried out with the Spectral Modeling, Analysis, Reduction Tool (SMART Ver. 5.5.1; Higdon et al. 2004). In the case of the low-resolution data though, the final reduced spectrum later used in this paper was retrieved from the recently published Cornell AtlaS of Spitzer IRS Sources (CASSIS; Lebouteiller et al. 2011a), which provided us with an improved and updated processing of the data.

In brief, the IRS data reduction starts from the intermediate pipeline products (droop files), which only lack stray light and flat-field correction. Individual pointings to each nod position of the slit were co-added using median averaging. For SL and LL spectra, we considered the difference of the two nod positions to remove the contribution of the background. Then, we extracted the spectra for each nod position using a variable width aperture, which scales the extraction aperture with wavelength to recover the same fraction of the diffraction limited instrumental pointspread function (PSF). The data from SH were extracted using the full slit extraction method from the median of the combined images. Since no sky (off-position) measurements were taken for the high-resolution module, the contribution of the sky emission was not subtracted from SH spectra. Then, the spectra were flux calibrated by multiplication with the relative spectral response function (RSRF), which was created from the IRS standard stars $\alpha$ Lac for SL and LL and $\xi$ Dra for SH, for which accurate templates were available (Cohen et al. 2003). We built our RSRFs by extracting the spectra of the calibration stars in the exact same way as the science target and dividing the stellar templates by the extracted stellar spectra. We produced one 


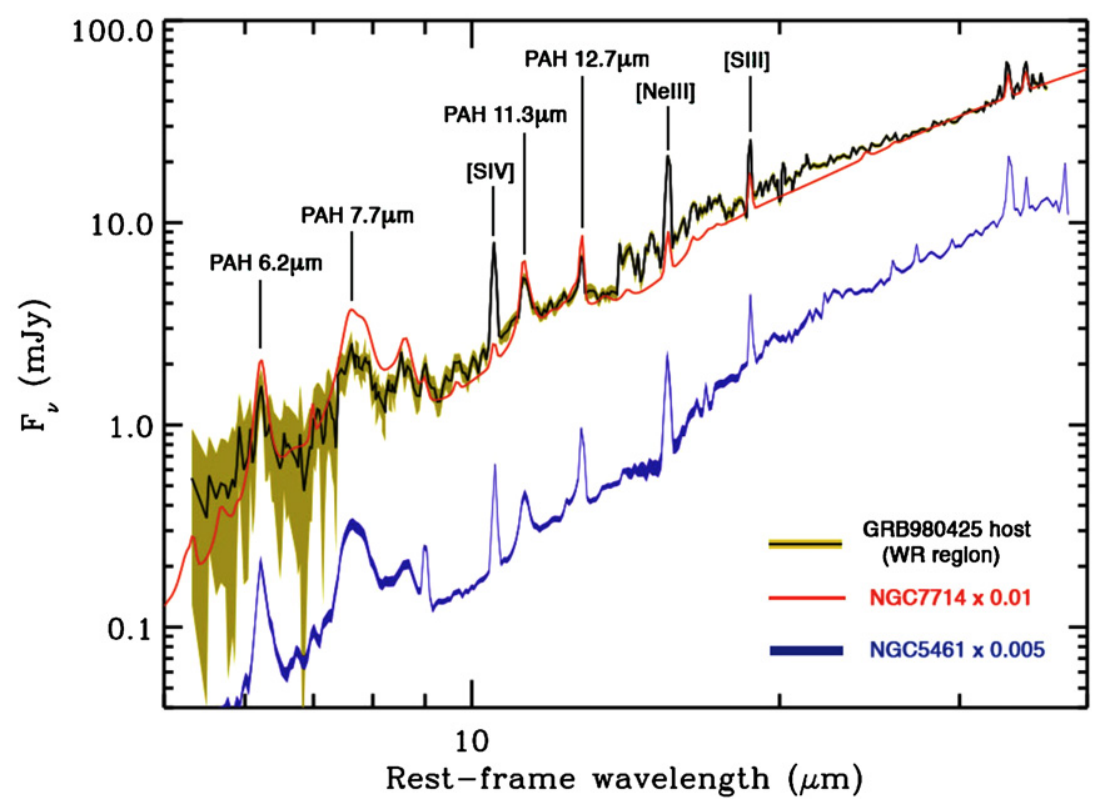

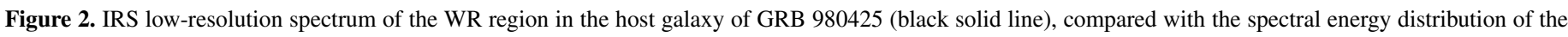

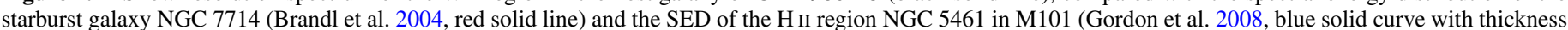

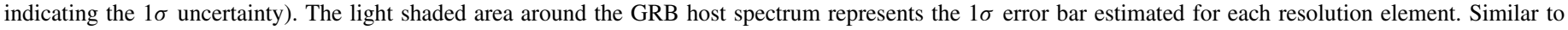
NGC 7714 and NGC 5461 the WR region exhibits a steeply rising continuum and bright PAH features, but also quite prominent ionic emission lines.

(A color version of this figure is available in the online journal.)

RSRF for each nod position in order to avoid systematic flatfield errors. The signal difference between the nod positions provide the error estimates. Finally, the flux-calibrated spectra of each order of the low-resolution modules were scaled to the first order of LL (LL1, 20-36 $\mu \mathrm{m}$ ), which was used to define the overall continuum of the source.

The final low-resolution IRS spectrum of the WR region is shown in Figure 2. Given the star-forming nature of GRB host galaxies, we compared it with the IRS observations of the starburst galaxy NGC 7714 (Brandl et al. 2004) and the superluminous H II region NGC 5461 in the spiral galaxy M101 (Gordon et al. 2008). The three SEDs exhibit striking similarities but also noticeable differences. First, they are all characterized by a steeply rising continuum of hot dust emission, and the polycyclic aromatic hydrocarbon (PAH) features commonly seen in star-forming galaxies (Laurent et al. 2000; Brandl et al. 2006; Smith et al. 2007) are also clearly apparent. These PAHs as well as the continuum emission originate from dust grains stochastically heated by the radiation field and by the UV photons from young populations of massive stars. Their luminosity correlates very well with the total IR luminosity measured between $8 \mu \mathrm{m}$ and $1000 \mu \mathrm{m}$ (Brandl et al. 2006) and they are therefore considered as a fairly accurate and extinctionfree tracer of the activity of star formation in galaxies (e.g., Roussel et al. 2001; Calzetti et al. 2007; Díaz-Santos et al. 2008).

However, we note that the $7.7 \mu \mathrm{m}$ PAH feature in the GRB host is substantially weaker than in NGC 7714 and NGC 5461, while the other main PAHs detected at $6.2 \mu \mathrm{m}, 11.3 \mu \mathrm{m}$, and $12.7 \mu \mathrm{m}$ have roughly the same relative strengths in the three spectra. Furthermore, the mid-IR spectral slopes are almost identical between the WR region and NGC 7714, while the midIR spectrum of the H II region NGC 5461 seems to rise even more rapidly at the longest wavelengths. Finally, the IRS spectrum of the WR region reveals the presence of prominent fine-structure emission lines such as [S IV]-10.51 $\mu \mathrm{m}$, [Ne III]-15.55 $\mu \mathrm{m}$, and
[S III]-18.71 $\mu \mathrm{m}$, which are also detected in NGC 5461 but either absent or much fainter in NGC 7714. In the following sections, we will quantitatively analyze the relative strengths of the different features characterizing the WR region of the GRB 980425 host. In particular, we will further explore how it differs from what has been so far observed in the mid-IR spectra of star-forming galaxies.

\section{MEASURED QUANTITIES}

\subsection{MIPS Broadband Photometry}

This section describes the broadband photometry measurements obtained from our MIPS imaging. All the measured fluxes are summarized in Table 1.

\subsubsection{MIPS $70 \mu \mathrm{m}$}

As one can see from Figure 1 the host of GRB 980425 is clearly detected at $70 \mu \mathrm{m}$, and in spite of the modest spatial resolution of the data, low surface brightness emission can also be observed up to a distance of $\sim 35^{\prime \prime}$ from the central region of the galaxy. To determine the total $70 \mu \mathrm{m}$ flux density of the host, we first estimated the sky background in the image by taking the median value of the pixels in the outskirt regions up to $\sim 100^{\prime \prime}$ from the center of the source. We then subtracted this sky level to each pixel of the mosaic and we measured the photometry inside a circular aperture with a diameter of $70^{\prime \prime}$ centered on the nucleus of the host. The photometric uncertainty was estimated from the pixel-to-pixel variations measured over the sky. We derived a total flux of $S_{70 \mu \mathrm{m}}=230 \pm 30 \mathrm{mJy}$.

Furthermore, the peak of the $70 \mu \mathrm{m}$ emission is clearly shifted from the center of mass of the host defined from the $4.5 \mu \mathrm{m}$ stellar emission, and there is strong evidence that the bulk of the $70 \mu \mathrm{m}$ luminosity originates from the WR region that also dominates the mid-IR luminosity of the galaxy. Figure 3 shows a larger field of view of the MIPS $24 \mu \mathrm{m}$ image of the GRB 980425 host, overlaid with the contours of the emission 
Table 1

Infrared Photometry and Global Properties of the WR Region and the GRB Host

\begin{tabular}{lccc}
\hline \hline & WR Region & GRB 980425 Host (Total) & Reference \\
\hline$F_{8 \mu \mathrm{m}}$ & $1.8 \pm 0.1 \mathrm{mJy}$ & $11.9 \pm 0.3 \mathrm{mJy}$ & Le Floc'h et al. $(2006)$ \\
$F_{24 \mu \mathrm{m}}$ & $19.5 \pm 2.0 \mathrm{mJy}$ & $26.2 \pm 1.3 \mathrm{mJy}$ & Section 3.1.3 \\
$F_{70 \mu \mathrm{m}}$ & $140 \pm 25 \mathrm{mJy}$ & $230 \pm 30 \mathrm{mJy}$ & Section 3.1 .1 \\
$F_{160 \mu \mathrm{m}}$ & $\ldots$ & $615 \pm 200 \mathrm{mJy}$ & Section 3.1 .2 \\
$\log \left(L_{\mathrm{IR}} / L_{\odot}\right)$ & $8.66 \pm 0.04$ & $9.01 \pm 0.07$ & Section 4.1 \\
$\mathrm{SFR}$ & $0.12 M_{\odot} \mathrm{yr}^{-1 \mathrm{a}}$ & $0.16 M_{\odot} \mathrm{yr}^{-1 \mathrm{~b}}$ & Section 4.2 \\
$\tau_{9.7 \mu \mathrm{m}}$ & 0.015 & $\ldots$ & Section 5 \\
\hline
\end{tabular}

Notes.

${ }^{a}$ Using the calibration from Bell et al. (2005).

${ }^{\mathrm{b}}$ Using the calibration from Calzetti et al. (2007).

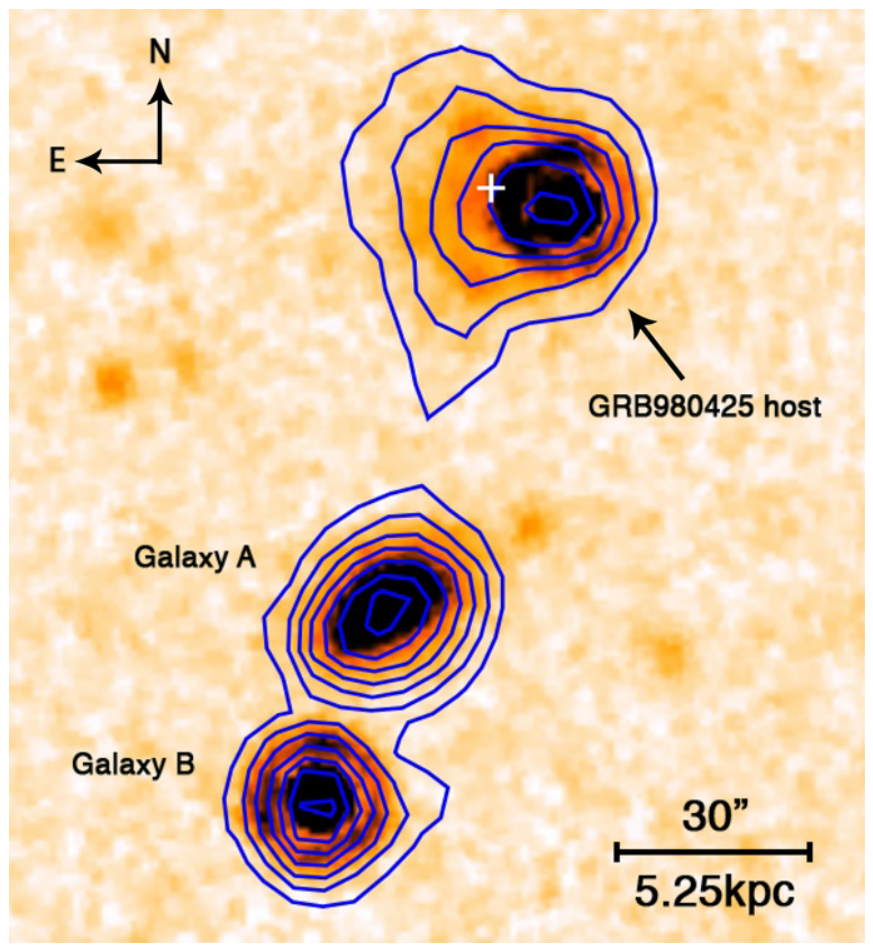

Figure 3. Surrounding of the GRB 980425 host galaxy imaged at $24 \mu \mathrm{m}$, superimposed with the contours of the $70 \mu \mathrm{m}$ emission. The GRB host is visible to the north while Galaxy A and Galaxy B identified by Foley et al. (2006) are also clearly detected at both wavelengths to the southeast. The contours scale linearly from 1.7 to $15 \mathrm{MJy} \mathrm{sr}^{-1}$. As in Figure 1, the white "cross" symbol (+) indicates the center of mass of the GRB host galaxy. In spite of the large FWHM of the far-IR MIPS PSF, the peak of the $70 \mu \mathrm{m}$ emission detected toward the host of GRB 980425 clearly coincides with the bright $24 \mu \mathrm{m}$ point source that corresponds to the WR region.

(A color version of this figure is available in the online journal.)

detected at $70 \mu \mathrm{m}$. We see that the peak of the $70 \mu \mathrm{m}$ emission coincides with the very bright $24 \mu \mathrm{m}$ point source lying in the southwest spiral arm at $12^{\prime \prime}$ from the center of the host galaxy.

Although this distance is slightly smaller than the FWHM of the MIPS $70 \mu \mathrm{m}$ PSF ( $\left.18^{\prime \prime}\right)$, we believe that the significance of this shift is robust given the high signal to noise of the $70 \mu \mathrm{m}$ emission detected toward the GRB host. As described in the next paragraph, the peak of the $70 \mu \mathrm{m}$ emission originates from a point source detected with a signal to noise $\mathrm{S} / \mathrm{N} \sim 5.6$. The positional $1 \sigma$ uncertainty associated with this point source can be estimated as $0.6 \times \mathrm{FWHM} \times(\mathrm{S} / \mathrm{N})^{-1}=6.75$ (see Appendix B of Ivison et al. 2007), which is twice smaller than the measured offset. Also, such a shift with respect to the nucleus of the galaxy could not be artificially produced by a misalignment between the $24 \mu \mathrm{m}$ and $70 \mu \mathrm{m}$ mosaics given the astrometric calibration of the Spitzer data $\left(1 \sigma \sim 1^{\prime \prime}\right.$ rms). In fact, there are two other galaxies detected to the south of the GRB host (see Figure 3) and their $70 \mu \mathrm{m}$ emission appears to be very well centered on the peak of their $24 \mu \mathrm{m}$ counterpart.

We compared the two-dimensional profile of the $70 \mu \mathrm{m}$ detection of the GRB 980425 host with the MIPS $70 \mu \mathrm{m}$ PSF. In spite of the lower spatial resolution of the MIPS data at this wavelength, we found that there is a spatially resolved low surface brightness component extending to the east of the peak of the $70 \mu \mathrm{m}$ emission. This component is observed over the whole extent of the galaxy and it is clearly apparent from the asymmetrical shape of the contours displayed in Figure 3 (note however that the small extension to the south comes from a data reduction artifact produced along one of the two dimensions of the $70 \mu \mathrm{m}$ pixel array). We thus decomposed the $70 \mu \mathrm{m}$ image of the GRB 980425 into a point source centered on the peak of the $70 \mu \mathrm{m}$ emission and the contribution from the spatially extended regions using the PSF-fitting routine of the DAOPHOT package (Stetson 1987). We found that the point source contributes to $140 \pm 25 \mathrm{mJy}$, which shows that a fraction as large as $\sim 60 \% \pm$ $10 \%$ of the $70 \mu \mathrm{m}$ emission of the GRB 980425 host originates from the WR region.

\subsubsection{MIPS $160 \mu \mathrm{m}$}

At $160 \mu \mathrm{m}$ the GRB 980425 host is also clearly detected in our data (Figure 1). However, the FWHM of the MIPS PSF at these wavelengths is comparable to the angular extent of the galaxy on the sky $\left(\sim 40^{\prime \prime}\right)$, and given the modest signal to noise of the observations it was not possible to confirm the presence of a spatially extended component similar to what we found in the $70 \mu \mathrm{m}$ image. The total flux density was therefore estimated with aperture photometry in a radius of $40^{\prime \prime}$, and a correction taking account of the PSF emission outside of this aperture was applied following the prescriptions described by Stansberry et al. (2007). We obtained a total flux of $S_{160 \mu \mathrm{m}}=615 \pm 200 \mathrm{mJy}$. Given the relatively small size of the $160 \mu \mathrm{m}$ pixel array as well as the contamination from the galaxy detected to the south of the GRB host (referred as Galaxy A by Foley et al. 2006, see also Figure 3), only a very small number of pixels were available to estimate the sky background in the image. This explains the large photometric uncertainty affecting our flux estimate.

Given the low angular resolution at these long wavelengths, it was not possible to perform a spatial decomposition similar to the one described above for the $70 \mu \mathrm{m}$ data. In particular, we could not determine the fraction of energy powered by the point source that dominates the monochromatic luminosity at shorter 


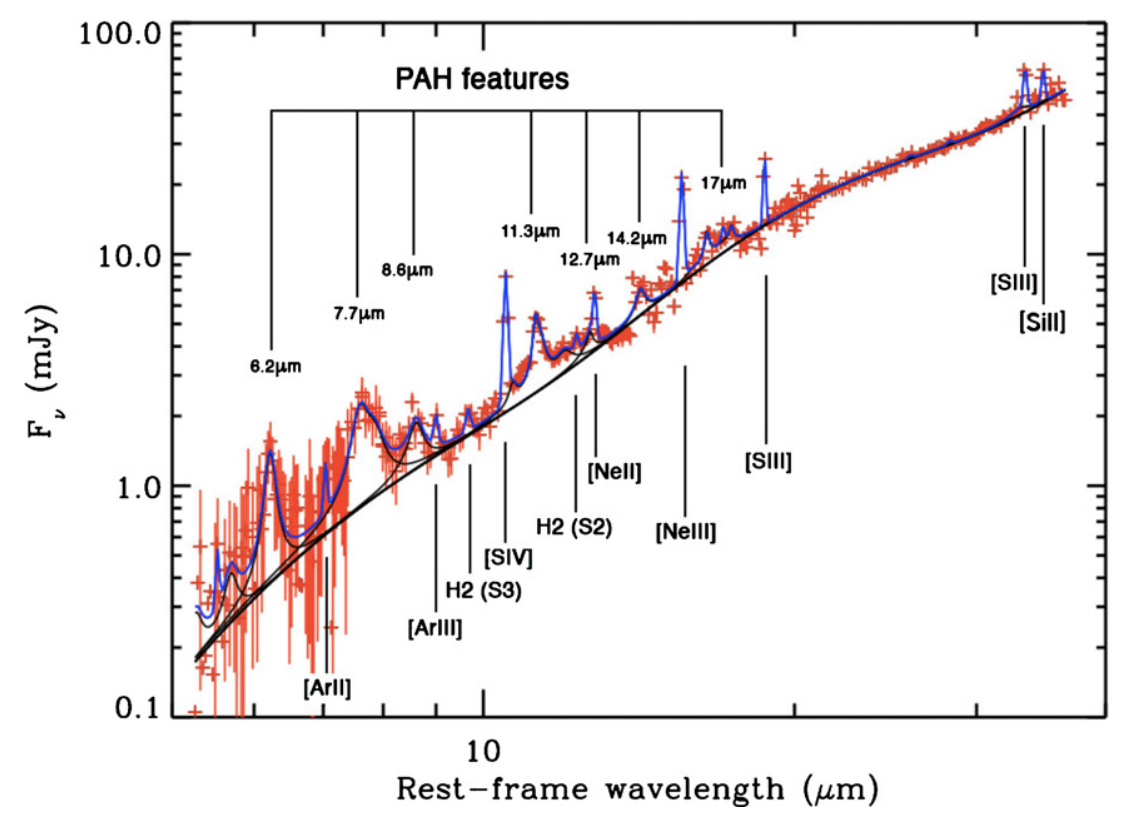

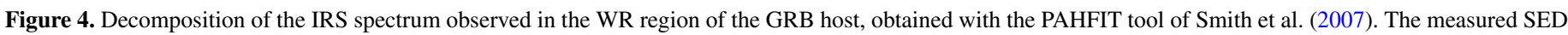

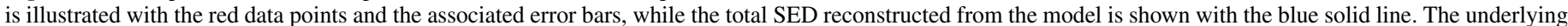

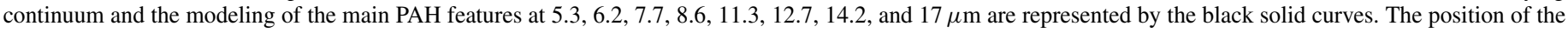
different ionic lines identified by the fit are also indicated.

(A color version of this figure is available in the online journal.)

IR wavelengths. However, Figure 1 reveals that the peak of the $160 \mu \mathrm{m}$ emission is again shifted to the southwest with respect to the center of the galaxy. Within the astrometric uncertainties it coincides with the peak of emission detected at $24 \mu \mathrm{m}$ and $70 \mu \mathrm{m}$, which suggests that the luminous $\mathrm{H}$ II region detected in the mid-IR is also responsible for a very large fraction of the total far-IR emission of the galaxy. ${ }^{12}$

\subsubsection{MIPS $24 \mu \mathrm{m}$}

The new $24 \mu \mathrm{m}$ image of the GRB 980425 host galaxy obtained in 2008 as part of this program looks very similar to the observations performed by MIPS/Spitzer in 2004 (Le Floc'h et al. 2006). Most of the emission originates from a single point-like source that appears to coincide with the WR region, while a couple of other $\mathrm{HII}$ regions as well as a more diffuse component are also detected toward the nucleus and in the spiral arms of the host. We measured the total flux of the galaxy and the luminosity of the WR region using a PSF-fitting decomposition and the same approach followed for the $70 \mu \mathrm{m}$ data. We found that the unresolved component contributes to a level of $19.5 \pm 2.0 \mathrm{mJy}$, while the total emission of the galaxy reaches $S_{24 \mu \mathrm{m}}=26.2 \pm 1.3 \mathrm{mJy}$. Within the error bars these results are fully consistent with the $24 \mu \mathrm{m}$ photometry of Le Floc'h et al. (2006). The lack of temporal variations shows that if some dust heating transient emission produced after the GRB explosion has contributed to the mid-IR emission of the galaxy, this emission must either be very faint (i.e., $\lesssim 10 \%$ of the total luminosity) or decrease very slowly with time.

\subsection{Mid-infrared Spectroscopic Features}

Numerous emission lines are clearly visible in the IRS spectroscopic observations of the WR region (Figure 2). They

\footnotetext{
12 The host of GRB 980425 is the only object covered by the field of view of the $160 \mu \mathrm{m}$ observations. Therefore, it was not possible to independently confirm the astrometry with other field galaxies as we did at $70 \mu \mathrm{m}$ and we had to rely on the Spitzer astrometric registration which is accurate to $1^{\prime \prime}$.
}

are commonly observed in the SED of star-forming galaxies and they provide valuable information on their activity of star formation as well as on the strength and the hardness of their radiation field. To constrain the luminosity of these features, we analyzed our low-resolution spectrum with the PAHFIT tool developed by Smith et al. (2007). This code was specifically designed for decomposing Spitzer IRS spectra into the contribution of the different components that characterize galaxy mid-IR SEDs. These are the hot dust continuum produced by the very small grains (VSGs), the broadband PAH features, the narrow ionic emission lines, and the effect of silicate absorption at $9.7 \mu \mathrm{m}$ and $18 \mu \mathrm{m}$. The result of our decomposition is displayed in Figure 4. Given the large number of individual features identified with PAHFIT, only the spectral profile corresponding to the main PAHs is represented, along with the shape of the underlying continuum (thick solid line). Similarly, only the position of the most prominent forbidden lines is labeled on the plot, and the total SED reconstructed from the model is shown as a blue solid line.

The narrow ionic lines observed between $10 \mu \mathrm{m}$ and $19 \mu \mathrm{m}$ are also clearly visible in the high-resolution spectrum that we obtained with IRS. A more accurate estimate of their luminosity was therefore obtained by fitting a Gaussian function to their emission profile derived from the $\mathrm{SH}$ data. This is illustrated in Figure 5, which shows the SH IRS observations obtained for the [S IV], [NeII], [Ne III], and [S III] ionic lines. Since no sky subtraction could be performed for these data though, the underlying continuum and the EWs of these features were estimated from the continuum of the low-resolution spectrum as modeled with PAHFIT. Our measurements are given in Table 2 along with the fluxes and the EWs of the main PAHs and ionic lines measured in the low-resolution data. For the strongest and isolated PAHs (i.e., $6.2 \mu \mathrm{m}, 7.7 \mu \mathrm{m}, 8.6 \mu \mathrm{m}$, and $11.3 \mu \mathrm{m}$ ), we indicate the results obtained with the global PAHFIT decomposition but we also provide the measures that we derived with a local fit of the continuum underlying each 

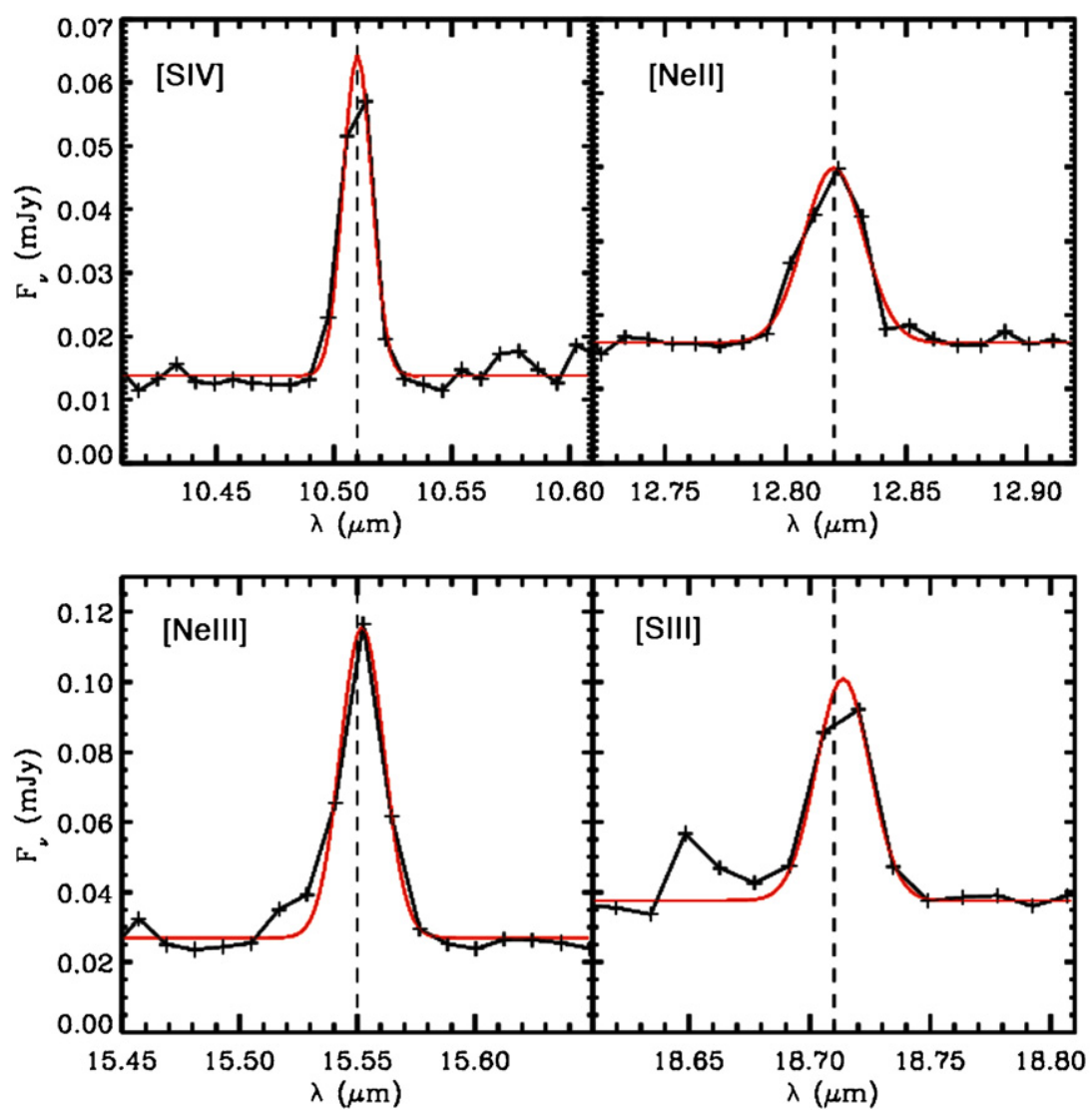

Figure 5. Close-up view of the profiles of the [S IV], [Ne II], [Ne III], and [S III] ionic lines observed with the IRS short-high module. In each panel, the observed data corrected for the redshift of the source are shown with the black ticked-solid curve while the model of the emission profile is performed with a Gaussian fit illustrated in red; the expected position of the forbidden line is indicated by the vertical dashed line.

(A color version of this figure is available in the online journal.)

Table 2

Mid-IR Emission Features

\begin{tabular}{|c|c|c|c|}
\hline Feature & $\begin{array}{c}\lambda \\
(\mu \mathrm{m})\end{array}$ & $\begin{array}{c}\text { Flux } \\
\times 10^{-21}\left(\mathrm{~W} \mathrm{~cm}^{-2}\right)\end{array}$ & $\begin{array}{l}\mathrm{EW} \\
(\mu \mathrm{m})\end{array}$ \\
\hline $\mathrm{PAH}^{\mathrm{a}}$ & 6.2 & $3.14 \pm 0.33(1.7 \pm 0.07)$ & $0.98(0.38)$ \\
\hline $\mathrm{PAH}^{\mathrm{a}}$ & 7.7 & $9.89 \pm 1.39(3.7 \pm 0.5)$ & $2.12(0.43)$ \\
\hline $\mathrm{PAH}^{\mathrm{a}}$ & 8.6 & $1.98 \pm 0.23(0.56 \pm 0.2)$ & $0.34(0.06)$ \\
\hline $\mathrm{PAH}^{\mathrm{a}}$ & 11.3 & $4.03 \pm 0.20(1.7 \pm 0.3)$ & $0.49(0.15)$ \\
\hline $\mathrm{PAH}^{\mathrm{b}}$ & 12.7 & $1.16 \pm 0.23$ & 0.12 \\
\hline $\mathrm{PAH}^{\mathrm{b}}$ & 17 & $7.58 \pm 0.75$ & 0.58 \\
\hline$[\mathrm{Ar} \text { II }]^{\mathrm{b}}$ & 7.01 & $0.18 \pm 0.16$ & 0.05 \\
\hline$[\mathrm{Ar} \text { III }]^{\mathrm{b}}$ & 9.01 & $0.19 \pm 0.08$ & 0.04 \\
\hline$\left[\mathrm{S}_{\mathrm{IV}}\right]^{\mathrm{c}}$ & 10.51 & $2.01 \pm 0.18$ & 0.40 \\
\hline $\mathrm{H} 2(\mathrm{~S} 2)^{\mathrm{c}}$ & 12.33 & $1.35 \pm 0.07$ & 0.22 \\
\hline$[\mathrm{Ne} \text { II }]^{\mathrm{c}}$ & 12.82 & $1.48 \pm 0.11$ & 0.23 \\
\hline$[\mathrm{Ne} I I I]^{\mathrm{c}}$ & 15.55 & $2.68 \pm 0.14$ & 0.34 \\
\hline$\left[\mathrm{S}_{\mathrm{III}}\right]^{\mathrm{c}}$ & 18.71 & $1.57 \pm 0.12$ & 0.16 \\
\hline$\left[\mathrm{S}_{\mathrm{IIII}}\right]^{\mathrm{b}}$ & 33.53 & $1.90 \pm 0.09$ & 0.17 \\
\hline$[\mathrm{Si} \text { II }]^{\mathrm{b}}$ & 34.86 & $1.44 \pm 0.09$ & 0.13 \\
\hline
\end{tabular}

Notes.

${ }^{a}$ Main values result from PAHFIT measurements in the low-resolution data. Values in parenthesis give another estimate independently obtained using a local spline-type fitting of the continuum.

${ }^{\mathrm{b}}$ Measured with the IRS low-resolution module using PAHFIT.

${ }^{c}$ Measured with the IRS high-resolution module, except for the continuum which was derived from the low-resolution data.

individual feature using a spline function. The latter approach has been commonly used in the literature to characterize the
mid-IR spectra of star-forming galaxies. It usually leads to lower values than obtained with PAHFIT, since PAHFIT accounts for the full extent of the PAH wings.

\section{TOTAL IR SEDs AND STAR FORMATION RATES}

\subsection{Infrared SED Fitting}

We constrained the SEDs of the GRB host galaxy and the WR region over the full infrared wavelength range by fitting the Spitzer broadband photometry with the empirical libraries of galaxy templates published by Chary \& Elbaz (2001), Dale \& Helou (2002), and Lagache et al. (2004), as well as with the physical SEDs derived from radiative transfer modeling by Siebenmorgen \& Krügel (2007). Between these different libraries, the SEDs mostly vary in the relative strength of the PAH features with respect to the hot dust continuum, as well as in the temperature and the emissivity of the cold dust component shaping the peak of the SED in the far-IR. In the library of Siebenmorgen \& Krügel (2007), the SEDs also depend on the size of the star-forming region responsible for the IR emission, and they are given for radii of $0.35,1,3,9$, and $15 \mathrm{kpc}$. We only considered sizes of $0.35 \mathrm{kpc}$ and $3 \mathrm{kpc}$ for the WR region and the whole galaxy, respectively.

To obtain the best possible constraints we combined the MIPS fluxes presented in Section 3.1 with the IRAC $8 \mu \mathrm{m}$ photometry already published by Le Floc'h et al. (2006). The fitting was performed separately for each library, using the code LePhare (Arnouts et al. 1999; Ilbert et al. 2006). Although most of the IR SED templates from the aforementioned libraries vary as a 


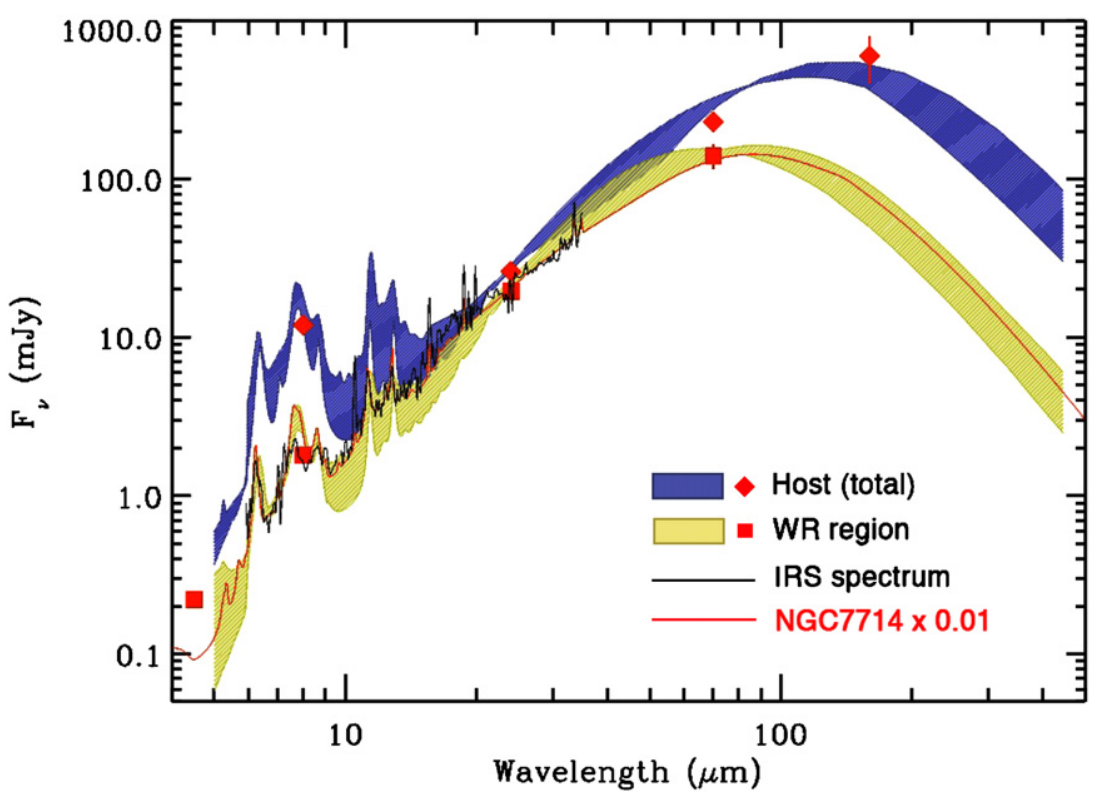

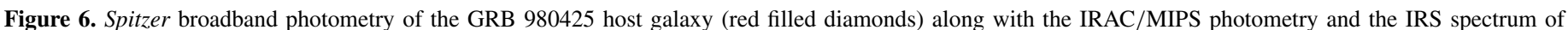

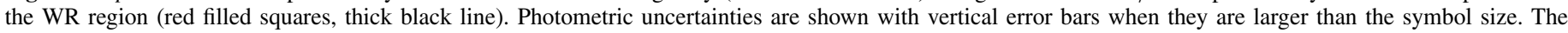

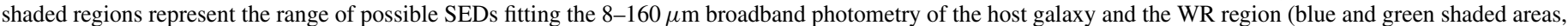

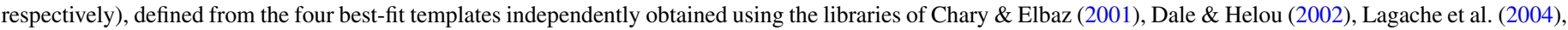

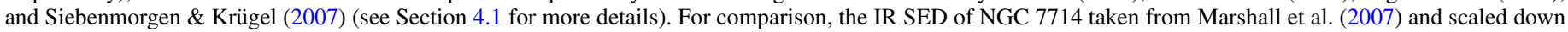
by a factor of 100 is also illustrated (thin red line).

(A color version of this figure is available in the online journal.)

function of the total IR luminosity (but see Dale \& Helou 2002 for a dependence on dust temperature), their normalization was kept as a free parameter and the best templates were derived from a basic $\chi^{2}$ minimization of the fit. In the case of the WR region, we did not include the photometry at $160 \mu \mathrm{m}$ since at this wavelength we were unable to separate its contribution from the emission of the host (see Section 3.1.2). We checked however that the best fits obtained for the WR region did not exceed the total flux measured for the GRB host galaxy at $160 \mu \mathrm{m}$.

The results are illustrated in Figure 6, which shows the measurements from our broadband photometry and IRS spectroscopy together with the global range of possible SED fits defined from the best-fit templates that were obtained for each of the four libraries. As expected from the different broadband fluxes measured with IRAC and MIPS, we note that the mid- to far-IR spectral slope of the WR region is much steeper than observed for the whole GRB host. By integrating the best fit SEDs between $8 \mu \mathrm{m}$ and $1000 \mu \mathrm{m}$, we derived total IR luminosities of $\log \left(L_{\mathrm{IR}} / L_{\odot}\right)=8.66 \pm 0.04$ and $\log \left(L_{\mathrm{IR}} / L_{\odot}\right)=9.01 \pm 0.07$ for the WR region and the whole GRB host galaxy, respectively. In these estimates, the uncertainties were obtained by combining (1) the dispersion measured between the four best fits obtained from the different libraries and (2) each of the dispersions measured within the individual libraries themselves. Our results thus suggest that $45 \% \pm$ $10 \%$ of the total IR luminosity of the GRB 980425 host galaxy originates from the WR region close to which the GRB was observed.

The lack of photometry at submillimeter wavelengths prevents us from fully constraining the Rayleigh-Jeans emission tail, which traces the properties of the cold dust component in the GRB host galaxy and the WR region. Therefore, the position of the IR SED peak and the characteristic temperature of the dust grain emission cannot unambiguously be determined with the present data. However, since we used different libraries of templates that significantly vary from one another, the results of our SED fitting suggest that we can reasonably predict the entire IR SED of both the GRB host and the WR region within an uncertainty of a factor of $\sim 2-3$. This can be explained by the fact that the IRAC/MIPS constraints imposed on the Wien side of the SED substantially reduced the number of possible fits from those libraries. In particular, we note that the SED of the WR region peaks at much shorter wavelengths than the SED of the whole galaxy (as also predicted by Michałowski et al. 2009 from stellar population synthesis modeling), showing that the characteristic temperature in the WR region is about twice larger than the average dust temperature in the ISM of the galaxy. This is also a direct consequence of the larger fraction contributed by the WR region at $24 \mu \mathrm{m}$ than at $70 \mu \mathrm{m}$.

\subsection{SFR Determination}

Based on the total IR luminosities computed in the previous section, we obtained a revised estimate of the SFRs in the WR region and the GRB host using the calibration provided by Bell et al. (2005): SFR $\left(M_{\odot} \mathrm{yr}^{-1}\right)=1.8 \times$ $10^{-10}\left(L_{\mathrm{IR}}+3.3 L_{2800}\right) / L_{\odot}$. In this relation, $L_{2800}$ represents the monochromatic luminosity $v L_{v}$ computed at $2800 \AA$, which we derived from a linear interpolation between the NUV and $U$-band fluxes published by Michałowski et al. (2009). In the case of the WR region, we obtained SFR $\sim 0.12 M_{\odot} \mathrm{yr}^{-1}$. Assuming the stellar mass $M_{*} \sim 5.7 \times 10^{6} M_{\odot}$ inferred by Michałowski et al. (2009), our estimate thus leads to a $\mathrm{SSFR} \sim 21 \mathrm{Gyr}^{-1}$, which corresponds to a typical timescale of $\sim 47 \mathrm{Myr}$ for this star-forming complex to double its mass.

Our new estimate of the SFR in the WR region is smaller than the one initially published by Le Floc'h et al. (2006) from the first Spitzer observations of the GRB 980425 host $\left(\mathrm{SFR} \sim 0.35 M_{\odot} \mathrm{yr}^{-1}\right)$, but this first determination had relied on a large extrapolation of the $24 \mu \mathrm{m}$ flux to infer the total IR 
luminosity characterizing this environment. Our revised SFR is in good agreement with that obtained by Michałowski et al. (2009) from broadband SED fitting $\left(\mathrm{SFR}=0.10 M_{\odot} \mathrm{yr}^{-1}\right)$, and it is also quite consistent with the values that can be inferred from the direct conversions recently established between the SFR and the mid-IR emission of galaxies (Alonso-Herrero et al. 2006; Calzetti et al. 2007; Díaz-Santos et al. 2008). For example, Alonso-Herrero et al. (2006) obtained a tight relationship between the $24 \mu \mathrm{m}$ and the extinction-corrected $\mathrm{Pa} \alpha$ luminosities of $\mathrm{H}$ II regions and star-forming galaxies in the local universe $\left(\mathrm{SFR} / M_{\odot} \mathrm{yr}^{-1}=8.45 \times 10^{-38}\left[L_{24 \mu \mathrm{m} / \mathrm{erg} \mathrm{s}}{ }^{-1}\right]^{0.871}\right)$, while Calzetti et al. (2007) established a comparable correlation between the SFR derived from the $\mathrm{H} \alpha$ recombination line and the $24 \mu \mathrm{m}$ emission of star-forming regions in nearby extragalactic sources. ${ }^{13}$ We applied these two relations to the $24 \mu \mathrm{m}$ flux of the WR region measured from our PSF fitting (see Section 3.1.3), which respectively led to SFR estimates of SFR $\sim 0.14 M_{\odot} \mathrm{yr}^{-1}$ and $\mathrm{SFR} \sim 0.13 M_{\odot} \mathrm{yr}^{-1}$. Considering the possible variations of the two calibrations with the metallicity and the age of the stellar populations ( $20 \%$; Calzetti et al. 2007), we find therefore a very good agreement with the revised estimate reported above from the UV and IR luminosities.

In the case of the entire host galaxy, the relations from Alonso-Herrero et al. (2006) and Calzetti et al. (2007) give a total SFR of $0.18 M_{\odot} \mathrm{yr}^{-1}$ and $0.16 M_{\odot} \mathrm{yr}^{-1}$, respectively, while the relation obtained by Bell et al. (2005) leads to a total $\mathrm{SFR} \sim 0.66 M_{\odot} \mathrm{yr}^{-1}$. At optical wavelengths though, the global SED of the galaxy is dominated by the contribution of passively evolving stellar populations (Savaglio et al. 2009) and it is likely that a large fraction of the total UV and IR luminosities of the host is not directly produced by ongoing or recent star formation. Consequently, the estimate of the SFR based on the relation from Bell et al. (2005) is probably overestimated.

\section{MID-INFRARED SPECTRAL PROPERTIES}

In Section 3.2, we used the PAHFIT tool to analyze the different components contributing to the IRS spectrum of the WR region (see Figure 4). Focusing first on the shape of the underlying VSG continuum, the decomposition reveals that there is almost no absorption from the silicate features at $9.7 \mu \mathrm{m}$ $\left(\tau_{9.7 \mu \mathrm{m}} \sim 0.015\right)$. Assuming $A_{V} / A_{9.7 \mu \mathrm{m}} \sim 20$ as observed in the Milky Way (Mathis 1990), the optical depth toward the silicates correspond to a small extinction $A_{V} \sim 0.3 \mathrm{mag}$. This is actually consistent with the modest obscuration that was derived toward the very center of the WR region based on the hydrogen Balmer decrement measured with Integral-Field Unit (IFU) spectroscopy ( $E(B-V) \sim 0.03$ mag; Christensen et al. 2008). It suggests that most of the activity in this area originates from a compact and optically thin environment and that the contribution from dust-enshrouded star formation must be very modest. Note that our estimate appears smaller than the value obtained by Hammer et al. (2006) using optical long-slit spectroscopy ( $\left.A_{V} \sim 1.5 \mathrm{mag}\right)$. Such differences could originate from aperture effects, since the extinction inferred by Christensen et al. (2008) reaches $E(B-V) \sim 0.17 \mathrm{mag}$ when it is measured over the entire WR region. It could also be due to the general difficulty in properly defining the underlying mid-IR spectral continuum around the $9.7 \mu \mathrm{m}$ silicate feature when prominent $7.7 \mu \mathrm{m}$ and $11.3 \mu \mathrm{m}$ PAHs are detected. This degeneracy in the mid-IR

\footnotetext{
13 Note that the relation inferred by Calzetti et al. (2007) needs to be corrected by a multiplicative factor of 1.59 to match the Salpeter IMF assumed in our analysis. See the Appendix A2 of Calzetti et al. (2007) for more details.
}

SED fitting results in additional uncertainties affecting the determination of the obscuration from the silicates, and it could have led to a small underestimate of the extinction in the case of the WR region.

Furthermore, the detection of prominent forbidden lines with high ionizing potential such as [Ne III], [S IV], or [Ar III] indicates the presence of a particularly hard radiation field. We quantified the ionizing conditions in the WR region using the lines fluxes measured from both the low- and high-resolution IRS spectra, which led to [Ne III]/[Ne II], [Ar III]/[Ar II], and $[\mathrm{S}$ IV] $/[\mathrm{S} \mathrm{III}]$ ratios of $1.8,1.05$, and 1.3 , respectively. Taking into account the sub-solar metallicity $\left(Z \lesssim 0.5 Z_{\odot}\right)$ inferred by Hammer et al. (2006) and Christensen et al. (2008), these ratios are comparable to what has been observed in other individually resolved $\mathrm{H}$ II regions and blue compact dwarfs (BCDs) with similar chemical abundances (Wu et al. 2006; Gordon et al. 2008; Hao et al. 2009). However, these values are substantially higher than what has been typically measured in star-forming and starburst galaxies (e.g., Brandl et al. 2006; Beirão et al. 2008), where the emission is likely averaged over different stellar populations spanning a wide range of properties and ages. Assuming that the mid-IR emission of the WR region originates from the central star cluster that produces most of the optical light (Hammer et al. 2006), the spatial extent of the $B$-band emission (i.e., $\lesssim 100 \mathrm{pc}$ ) and the hardness of the radiation field derived with IRS suggest that most of the mid-IR luminosity is produced by very young massive star formation. Following Rigby \& Rieke (2004), who studied the evolution of various midIR line ratios with time using the CLOUDY photoionization model, we find that the $[\mathrm{Ne} I I I] /[\mathrm{NeII}],[\mathrm{Ar} I I I] /[\mathrm{Ar} I \mathrm{I}]$, and [S IV]/[S III] ionizing indices measured in the GRB 980425 host are compatible with a starburst episode younger than $\sim 5 \mathrm{Myr}$. This is consistent with the estimate obtained by Hammer et al. (2006) based on the number of WR stars observed in this region (i.e., 1-6 Myr).

The mid-IR emission line diagnostics that can be used to characterize the ionizing conditions of star-forming environments are obviously not independent from each other. A number of correlations have been established between [ $\mathrm{Ne} \mathrm{III}] /[\mathrm{Ne} \mathrm{II}]$, [Ar III]/[Ar II], and [S IV] /[S III], as well as with [S IV]/[Ne II] and with optical line ratios like $[\mathrm{O}$ III $] \lambda 5007 /[\mathrm{O}$ II $] \lambda 3727$ (e.g., Giveon et al. 2002; Wu et al. 2006; Dale et al. 2006; Groves et al. 2008; Gordon et al. 2008; Hao et al. 2009; BernardSalas et al. 2009). The mid-IR spectral properties of the WR region are fully consistent with these different relationships as well as with the rough correlation that exists between the metallicity of galaxies and the hardness of their radiation field (Wu et al. 2006; Gordon et al. 2008). Moreover, the electron density in the WR region can be constrained from the two Sulfur III emission lines detected with IRS. The flux ratio $[\mathrm{S}$ III $] \lambda 18.71 /\left[\mathrm{S}_{\mathrm{III}}\right] \lambda 33.53 \sim 0.8$ corresponds to $N_{e} \sim 200 \mathrm{~cm}^{-3}$ (Giveon et al. 2002). This is slightly larger than the estimate that was derived from the optical spectrum of the WR region using the $[\mathrm{S}$ III] $]$ 6716/[S III] 66731 flux ratio $\left(N_{e}=158 \mathrm{~cm}^{-3}\right.$; Hammer et al. 2006) and it is also higher than the densities measured in the HII regions of M101 (S III] 18.71 / [S III] $333.53 \sim 0.2$; Gordon et al. 2008). This could be related to the effect of the stellar winds produced by the WR populations, which may have efficiently compressed the gas and the ISM of the WR region in the host of GRB 980425.

Finally, the EWs of the PAH features characterizing the WR region fall within expectations given the metallicity and the hardness of the radiation field previously measured. 
In star-forming galaxies the relative strength of PAHs over the VSG continuum is known to remain constant over a wide range of environments, but it rapidly decreases with harder ionizing radiations. This is particularly apparent when the hardness of the radiation field reaches a threshold of [Ne III $] /[\mathrm{Ne}$ II $] \sim 1-3$ (Gordon et al. 2008; Lebouteiller et al. 2011b) or similarly when the chemical abundance gets lower than $12+\log [\mathrm{O} / \mathrm{H}] \sim$ 8-8.3 (e.g., Houck et al. 2004a; Engelbracht et al. 2005; Wu et al. 2006; Engelbracht et al. 2008). The properties of the WR region in the GRB 980425 host galaxy correspond to this regime of transition between extreme metal-poor regions and environments with solar metallicity, and the PAHs are clearly detected albeit with smaller EWs than observed in sources with softer radiation fields. Looking at Figure 2, we also note that the $7.7 \mu \mathrm{m} / 11.3 \mu \mathrm{m}$ PAH inter-band ratio is substantially smaller than observed in the mid-IR spectrum of the prototypical starburst galaxy NGC 7714 but it is once again in agreement with the trend that has been seen between this quantity and the ionizing index measured with the $[\mathrm{Ne}$ III $] /[\mathrm{Ne}$ II] line ratio (Smith et al. 2007). Given that the $11.3 \mu \mathrm{m}$ feature can be produced by larger PAHs than the ones responsible for the $7.7 \mu \mathrm{m}$ emission (Draine \& Li 2007), the WR region could thus be experiencing a selective destruction of the small PAHs that emit at short mid-IR wavelengths (Smith et al. 2007).

\section{DISCUSSION}

\subsection{Comparison with Other Known Extragalactic Sources}

The total IR luminosity derived in Section $4\left(\log \left[L_{\mathrm{IR}} / L_{\odot}\right] \sim\right.$ 8.7) places the WR region at the very bright end of the luminosity function of $\mathrm{H}$ II regions observed in the local universe (Bradley et al. 2006; Lee et al. 2011). Considering the physical size of the optical light distribution measured in the HST image of the host ( $\lesssim 100$ pc; Fynbo et al. 2000; Hammer et al. 2006), this environment is also one of the single-isolated $\mathrm{H}$ II regions with the highest star formation density identified so far. For instance, the brightest star-forming complex in the Milky Way, W49A, releases up to $L_{\mathrm{IR}} \sim 2.7 \times 10^{7} L_{\odot}$ within a size less than $\sim 50$ pc in diameter (Ward-Thompson \& Robson 1990), while the total luminosity of the 30 Doradus nebula in the Large Magellanic Cloud only reaches $L_{\mathrm{IR}} \sim 4 \times 10^{7} L_{\odot}$ (Werner et al. 1978). However, some of the intrinsic characteristics of this environment have already been observed in other extragalactic sources, which suggest that such a complex of star formation does not represent a unique object in the local universe. For instance, isolated $\mathrm{H}$ II regions with similar mid-IR spectral slope and ionization indices have been found in the giant spiral galaxy M101 (Gordon et al. 2008). Among these regions, NGC 5461 does contain a population of WR stars (Schaerer et al. 1999) and it has a $24 \mu \mathrm{m}$ flux of $\sim 920 \mathrm{mJy}$, which given the distance of M101 (6.7 Mpc) corresponds to a mid-IR luminosity 50\% larger than what we measured in the host of GRB 980425. The mid-IR properties of the WR region also resemble what is seen in BCD galaxies with comparable oxygen abundance, which often exhibit a steeply rising hot dust continuum with prominent ionic lines and very little absorption by the silicate features (Wu et al. 2006). Similarly, some of the giant super star clusters in the overlap region of the Antennae exhibit comparable or steeper mid-IR continuum emission as well as higher SFRs, although these clusters may be spatially more extended and their radiation field is typically a bit softer than observed in the WR region (Brandl et al. 2009).

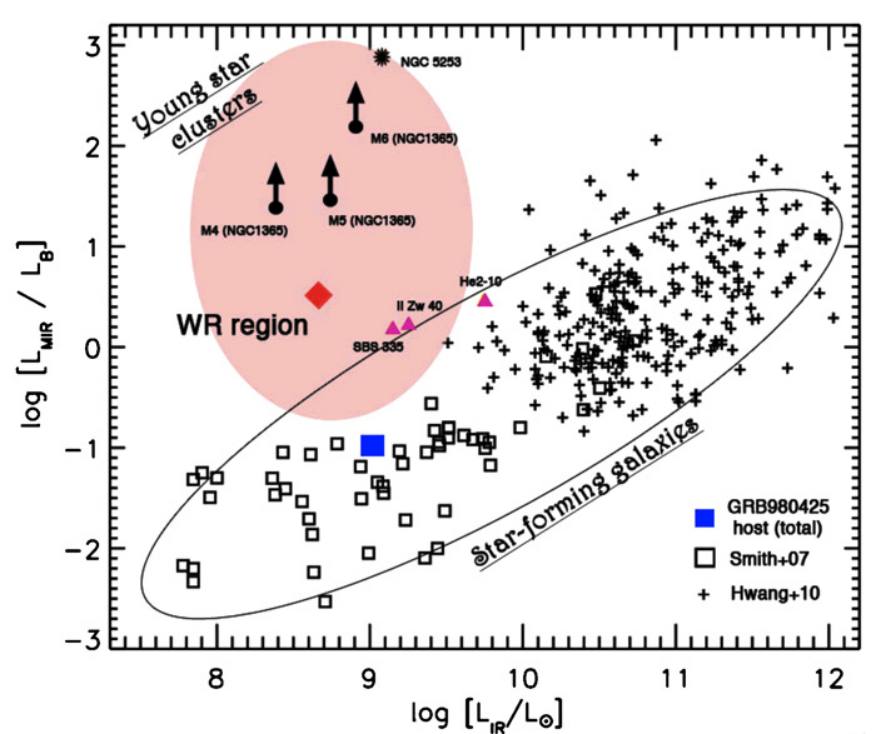

Figure 7. Mid-IR over $B$-band monochromatic luminosity ratio $\left(L_{\mathrm{MIR}} / L_{B}\right)$ plotted against the total IR luminosity of the WR region (red filled diamond) and the entire GRB 980425 host galaxy (blue filled square). For comparison, we also indicate the local star-forming galaxy samples of SINGS (open squares; Smith et al. 2007) and SDSS ("+" symbols; Hwang et al. 2010), the prototypical blue compact dwarfs SBS 0335-052, II Zw 40, and He2-10 (pink triangles; Hunt et al. 2005), the embedded starburst in NGC 5253 ("*" symbol; Alonso-Herrero et al. 2004), and the young star clusters of NGC 1365 (lower limits; Galliano et al. 2008). Mid-IR luminosities were computed based on MIPS-24 $\mu \mathrm{m}$ fluxes except for the SDSS sample where the IRAS $25 \mu \mathrm{m}$ bandpass was used as a proxy. The $L_{\mathrm{MIR}} / L_{B}$ ratio in the WR region is much higher than typically found in star-forming galaxies with comparable luminosities, but it is lower than observed in young star clusters still embedded in clouds of dust and molecular gas.

(A color version of this figure is available in the online journal.)

What is nonetheless very unusual in the host of GRB 980425 is the large fraction $(45 \% \pm 10 \%)$ of the total infrared luminosity of the galaxy contributed by the WR region alone. This large contribution to the global star-forming activity of the host is not apparent at optical wavelengths, because the ratio of the mid-IR and optical luminosities in the WR region is much higher than measured over the whole spatial extent of the galaxy. This is illustrated in Figure 7, which shows the $24 \mu \mathrm{m}$ over $B$-band monochromatic luminosity ratio $L_{\mathrm{MIR}} / L_{B}=$ $\left(v L_{v}\right)_{24 \mu \mathrm{m}} /\left(v L_{v}\right)_{B}$ of the host and the WR region, plotted as a function of their total Infrared luminosity. In both cases, the $B$-band luminosities were taken from Michałowski et al. (2009). For comparison we over plotted the ratios derived from the Sloan Digital Sky Survey (SDSS) by Hwang et al. (2010), those measured by Dale et al. (2006) and Smith et al. (2007) in the Spitzer Infrared Nearby Galaxies Survey (SINGS), and the ratios characterizing the three BCD prototypes SBS 0335-052, II Zw 40, and He2-10 (Hunt et al. 2005). While the $L_{\mathrm{MIR}} / L_{B}$ integrated over the host of GRB 980425 is typical of quiescent star-forming galaxies with similar luminosities, we see that the ratio in the WR region is more than an order of magnitude higher, and comparable to that observed in BCDs.

This characteristic of the WR region is actually consistent with what can be observed in recent starbursts, despite the apparently modest extinction derived from the $9.7 \mu \mathrm{m}$ silicate features and the hydrogen Balmer decrement (see Section 5). In fact, very young and dust-embedded stellar clusters with much higher extinction (e.g., $A_{V} \gtrsim 5 \mathrm{mag}$ ) can harbor $L_{\mathrm{MIR}} / L_{B}$ ratios even larger than found in the WR region. As an example we included in Figure 7 the case of the "hypernebula" starburst enshrouded in the core of NGC 5253 (Alonso-Herrero et al. 
2004) as well as the massive star clusters identified in NGC 1365 by Galliano et al. (2008). These complexes of star formation share strong similarities with the WR region in the GRB 980425 host in terms of SFR, age, and luminosities. However, the optical depth toward these sources is much more significant, explaining their extreme optical-to-infrared colors. Assuming that the obscuration and the IR/optical luminosity ratio decrease with the starburst age, what we are seeing in the WR region could thus be one or several star clusters that have already evolved and partly escaped the original molecular cloud where they were born. Alternatively, and rather than an "aging" effect, the difference of visual extinction between the WR region of the GRB 980425 host and the enshrouded clusters of NGC 5253 and NGC 1365 could also be a direct consequence of the presence of the WR stars themselves, which may have accelerated the process of clearing out the local ISM of the star-forming region via their emission of long-lasting supersonic winds. Depending on geometry effects, such a scenario could lead to small silicate absorption as observed, while a still important emission of very hot dust at mid-IR wavelengths could arise from amorphous carbon grains heated to very high temperatures by the massive stars dominating the spectrum of the WR region.

\subsection{Long Gamma-Ray Bursts and Superluminous H II Regions}

As demonstrated in Section 4, the most striking result of our analysis resides in the bolometric luminosity of the GRB 980425 host, half of which originates from a single and compact starforming complex located at more than $2 \mathrm{kpc}$ from the galaxy center. This large contribution of extranuclear star formation has also been observed in a few other spiral galaxies of the local universe (e.g., NGC 5257, NGC 6670, Arp 256; Haan et al. 2011), although these cases are rare and they have much higher mass and SFR than the host of GRB 980425. Most often, the star-forming activity of morphologically evolved systems in the luminosity range of LGRB hosts appears to be preferentially distributed among a large number of $\mathrm{H}$ II regions or centrally concentrated toward ring-like structures of multiple knots and star clusters. The fact that this atypical configuration is found in a galaxy initially selected as the host of a rare event such as a GRB therefore raises the question of a possible link between the physical properties of the WR region and the trigger of the LGRBs in star-forming galaxies.

Indeed, the properties observed in this environment look similar to a number of specific characteristics commonly found in GRB host galaxies at cosmological distances. First, the midIR spectroscopic features and the SSFR of the WR region both confirm the presence of extremely young star formation (i.e., $<5 \mathrm{Myr}$ ) in this area. Assuming a constant SFR, the SSFR that we derived in Section 4.2 indicates that this complex of massive star formation would double its stellar mass in only $47 \mathrm{Myr}$. This is extremely short compared to the typical timescale of galaxy evolution, and although it is not as extreme in other GRB host galaxies it is consistent with the higher SSFR displayed by this population when compared to field star-forming galaxies at similar redshifts (Christensen et al. 2004; Thöne et al. 2008). Second, the WR region has sub-solar metallicity and only modest extinction by dust and therefore very blue optical colors (Fynbo et al. 2000; Hammer et al. 2006; Christensen et al. 2008; Michałowski et al. 2009) similar to what is typically observed for GRB hosts at higher redshifts (Fruchter 1999; Le Floc'h et al. 2003; Han et al. 2010). Among the numerous H II environments detected in the GRB 980425 host it also harbors the largest surface brightness region of the galaxy, which is consistent with the link that was established by Fruchter et al. (2006) between the location of GRBs and the very brightest regions of the hosts. Finally, optical emission line ratios like $[\mathrm{O} \mathrm{III}] / \mathrm{H} \beta,[\mathrm{N} \mathrm{II}] / \mathrm{H} \alpha$, $[\mathrm{S}$ II $] / \mathrm{H} \alpha$, and $[\mathrm{N} \mathrm{II}] /[\mathrm{O}$ II $]$ suggest that the ionizing conditions and the stellar populations in the WR region are very similar to that observed in more distant and unresolved GRB host galaxies (Christensen et al. 2008). Without claiming a direct link between the birth of the GRB 980425 progenitor and the WR region, it would seem therefore difficult from all of these observations to argue against a kind of causal link between the two.

Besides, and contrary to the characteristics of the WR region, it is worth noting that the integrated properties of the GRB 980425 host galaxy differ from those of other GRB hosts at high redshift. For example, the host of GRB 980425 is an already-evolved galaxy that is still forming stars, but at a very low rate with respect to the stellar mass that it has already built (Savaglio et al. 2009; Michałowski et al. 2009). Furthermore, the $[\mathrm{O} \mathrm{III}] / \mathrm{H} \beta$ line ratio measured in the other $\mathrm{H}$ II regions of the GRB 980425 host are substantially smaller than what is typically observed in more distant LGRB host galaxies (Christensen et al. 2008). These differences further emphasize the connection that must exist between the episode of star formation currently taking place in the WR region and the trigger of the GRB 980425 in this galaxy.

Nonetheless, the most unsettling fact is that GRB 980425 did not occur within the WR region but at a projected distance $\sim 900$ pc away. What can then be the physical connection between the two? LGRBs represent extremely rare phenomena that are more likely to occur from large populations of massive stars. Given the very low density of the latter in the area where GRB 980425 was found, and considering the presence of numerous WR and OB stars in the WR region, Hammer et al. (2006) suggested a "runaway scenario" in which the GRB 980425 progenitor may have come from the WR region itself. This progenitor would have been dynamically ejected after a kick or close stellar encounters, and traveled at velocities $\sim 200-300 \mathrm{~km} \mathrm{~s}^{-1}$ across the ISM of the galaxy before ending its life as a GRB. Very massive runaway stars with such velocities are actually expected from numerical simulations of dynamical encounters in stellar clusters (e.g., Gvaramadze \& Gualandris 2011). In the mid-infrared, the detection of very high excitation transition lines such as [O IV] at $25.9 \mu \mathrm{m}$ can indicate the presence of very hot WR stars (Schaerer \& Stasińska 1999) or recent supernovae, which in the case of the WR region could further strengthen this possible connection with the progenitor of GRB 980425. In the IRS spectrum, however, the emission from [O IV] is only observed at the $2 \sigma$ level, making this association uncertain.

Conversely, star formation is still ongoing at the location where GRB 98425 was observed and therefore one cannot fully rule out that its progenitor was truly born in this area. For instance, this site could have experienced several million years ago a massive starbursting episode strongly biased toward the high mass end of the IMF, hence explaining the relatively low density of stars now observed in this area compared to that of the WR region. In this second scenario, it is the physical mechanism that triggered star formation at both locations, as well as similarities between the chemical properties of these two environments, that would explain a possible relationship between the WR region and GRB 980425. For instance, a single density wave propagating through the disk of the galaxy could have triggered over a short timescale but in a sequential way the formation of new stellar populations along the southeastern 
spiral arm, and we would be seeing the host while the star-forming episode at the GRB location has just terminated. In fact, internal processes must have played a critical role in driving the recent star-forming activity in this object. The galaxy ESO 184-G82 appears as an isolated system in the sky (Foley et al. 2006) and a trigger of star formation due to the accretion of external material via minor merging seems quite unlikely.

\subsection{Perspectives}

The current data are unfortunately not sufficient to determine the exact origin of the progenitor of GRB 980425. Further progress may be expected from IFU kinematic studies, which could provide better constraints on the velocity fields and the dynamical properties of the stellar populations in the host galaxy. High-resolution maps of the molecular gas distribution inferred from facilities like the Atacama Large Millimeter Array (ALMA) would also help constraining how the star formation efficiency varies in the surrounding of the WR region.

Similarly, characterizing with more statistically representative samples the locations of GRBs with respect to the distribution of star-forming regions in their hosts should provide more insights into the connection between the occurrence of LGRBs and the dynamical ejection of massive stars in stellar clusters. A clear trend has already been observed by Fruchter et al. (2006) between the locations of cosmological GRBs and the brightest regions of their hosts (see also Svensson et al. 2010). However, the spatial resolution of current facilities has not yet been sufficient to constrain possible offsets at sub-kpc scales. Further improvements should thus be expected from next generation instruments like the James Webb Space Telescope and the Extremely Large Telescopes.

Independently, the properties of the WR region in the host of GRB 980425 reinforce the idea that LGRBs are triggered within or in the vicinity of extremely young but active regions of massive star formation, lending further support to their collapsar origin (MacFadyen \& Woosley 1999). Similar to what can be inferred from the chemical abundance of the WR region (Hammer et al. 2006; Christensen et al. 2008) this star-forming activity tends to be associated with metal-poor environments, hence explaining that LGRBs rather occur within relatively unobscured blue galaxies with sub-solar oxygen abundances (Han et al. 2010).

\section{SUMMARY AND CONCLUSIONS}

Thanks to its relatively small distance (i.e., $36 \mathrm{Mpc}$; Galama et al. 1998), the host of GRB 980425 represents one of the very few cases of LGRB hosts where spectroscopic and bolometric properties can be spatially resolved. It thus offers a unique opportunity to constrain the characteristics of the close environment where LGRBs take place. In this work, we present Spitzer 5-160 $\mu \mathrm{m}$ spectro-imaging data of the GRB 980425 host galaxy and the "Wolf-Rayet region" close to which the GRB was observed. Our main results can be summarized as follows.

1. Given the compactness of the WR region $(<100 \mathrm{pc}$ in diameter), the highly ionized emission lines in our IRS spectrum (e.g., [Ne III], [S IV], [Ar III]) suggest that this environment is associated with extremely young ( $<5 \mathrm{Myr})$ massive star formation.

2. While the whole GRB 980425 host galaxy is producing stars at a very low rate compared to its already-formed stellar populations, the WR region will double its stellar mass in only $47 \mathrm{Myr}$ assuming constant SFR. It has a total
IR luminosity of $\sim 4.6 \times 10^{8} L_{\odot}$ and it represents one of the most luminous $\mathrm{H}$ II regions identified in the nearby universe.

3. In the WR region, the relative strength of the PAH features over the continuum is similar to what has been observed in other systems with comparable oxygen abundance $(Z \lesssim$ $\left.0.5 Z_{\odot}\right)$. The extinction measured from the optical depth of the $9.7 \mu \mathrm{m}$ silicate feature implies modest obscuration $\left(\tau_{9.7 \mu \mathrm{m}} \sim 0.015\right)$.

4. Although the WR region produces less than $5 \%$ of the $B$-band emission of the GRB host, it contributes up to $45 \% \pm 10 \%$ of the total IR luminosity of the galaxy. Its IR SED also peaks at much shorter wavelengths than the global SED of the host, suggesting that the characteristic temperature in the WR region is about twice that of the average dust temperature in the ISM of the system.

5. The ratio between the mid-IR and the $B$-band luminosities in the WR region is characteristic of recent starbursts and much higher than what is observed in star-forming galaxies with similar $L_{\mathrm{IR}}$. However, it is not as extreme as in very young and dust-embedded stellar clusters that harbor much stronger extinction than derived from the mid-IR spectrum of the GRB host. Given the blue colors of the WR region at optical wavelengths, this suggests that this complex of star formation harbors one or several star clusters that have already evolved and partly escaped the original molecular cloud where they were born.

The contribution of the WR region to the bolometric output of the GRB 980425 host makes this galaxy a rather unique object of the nearby universe. Considering that GRBs represent extremely rare events, our analysis supports therefore the existence of a causal link between the origin of GRB 980425 and the activity of star formation in the WR region. More generally, and also taking account of the properties characterizing the whole population of LGRB hosts, our results corroborate the common idea that the triggering of LGRBs is mostly associated with very active star formation in chemically young environments.

This work was enabled based on the funding from the IRS and the MIPS projects which are supported by NASA through the Jet Propulsion Laboratory (subcontracts 1257184 and 960785), and thanks to the efficient technical support provided by the Spitzer Science Center. We thank our referee for relevant suggestions and a careful review of the manuscript as well as Michal Michałowski for useful comments on our work. We are particularly grateful to Vianney Lebouteiller for fruitful discussions and for providing us with updated IRS data reduction prior to the publication of the Cornell AtlaS of Spitzer IRS Sources (CASSIS; Lebouteiller et al. 2011a) and to Ho Seong Hwang for sharing his results on the SDSS data.

We also acknowledge Yanling Wu for her help in the IRS data reduction as well as Jason Marshall for providing some of his IR SED modeling and J.D. Smith for making publicly available his PAHFIT routines. We greatly appreciated the help from Stephane Arnouts and Olivier Ilbert when using the code Le Phare. We finally thank Tanio Díaz-Santos, Frédéric Galliano, David Elbaz, Suzanne Madden, and Marc Sauvage for useful discussions.

\section{REFERENCES}

Alonso-Herrero, A., Pérez-González, P. G., Alexander, D. M., et al. 2006, ApJ, 640,167

Alonso-Herrero, A., Takagi, T., Baker, A. J., et al. 2004, ApJ, 612, 222 
Arnouts, S., Cristiani, S., Moscardini, L., et al. 1999, MNRAS, 310, 540 Beirão, P., Brandl, B. R., Appleton, P. N., et al. 2008, ApJ, 676, 304 Bell, E. F., Papovich, C., Wolf, C., et al. 2005, ApJ, 625, 23

Bernard-Salas, J., Spoon, H. W. W., Charmandaris, V., et al. 2009, ApJS, 184 230

Bloom, J. S., Kulkarni, S. R., \& Djorgovski, S. G. 2002, AJ, 123, 1111

Bradley, T. R., Knapen, J. H., Beckman, J. E., \& Folkes, S. L. 2006, A\&A, 459, L13

Brandl, B. R., Bernard-Salas, J., Spoon, H. W. W., et al. 2006, ApJ, 653, 1129

Brandl, B. R., Devost, D., Higdon, S. J. U., et al. 2004, ApJS, 154, 188

Brandl, B. R., Snijders, L., den Brok, M., et al. 2009, ApJ, 699, 1982

Calzetti, D., Kennicutt, R. C., Engelbracht, C. W., et al. 2007, ApJ, 666, 870

Chary, R., \& Elbaz, D. 2001, ApJ, 556, 562

Christensen, L., Hjorth, J., \& Gorosabel, J. 2004, A\&A, 425, 913

Christensen, L., Vreeswijk, P. M., Sollerman, J., et al. 2008, A\&A, 490, 45

Cohen, M., Megeath, S. T., Hammersley, P. L., Martin-Luis, F., \& Stauffer, J. 2003, AJ, 125, 2645

Courty, S., Björnsson, G., \& Gudmundsson, E. H. 2004, MNRAS, 354, 581

Courty, S., Björnsson, G., \& Gudmundsson, E. H. 2007, MNRAS, 376, 1375

Daigne, F., \& Mochkovitch, R. 2000, A\&A, 358, 1157

Dale, D. A., \& Helou, G. 2002, ApJ, 576, 159

Dale, D. A., Smith, J. D. T., Armus, L., et al. 2006, ApJ, 646, 161

Díaz-Santos, T., Alonso-Herrero, A., Colina, L., et al. 2008, ApJ, 685, 211

Draine, B. T., \& Li, A. 2007, ApJ, 657, 810

Engelbracht, C. W., Gordon, K. D., Rieke, G. H., et al. 2005, ApJ, 628, L29

Engelbracht, C. W., Rieke, G. H., Gordon, K. D., et al. 2008, ApJ, 678, 804

Foley, S., Watson, D., Gorosabel, J., et al. 2006, A\&A, 447, 891

Fruchter, A. S. 1999, ApJ, 512, L1

Fruchter, A. S., Levan, A. J., Strolger, L., et al. 2006, Nature, 441, 463

Fynbo, J. P. U., Jakobsson, P., Möller, P., et al. 2003, A\&A, 406, L63

Fynbo, J. U., Holland, S., Andersen, M. I., et al. 2000, ApJ, 542, L89

Galama, T. J., Vreeswijk, P. M., van Paradijs, J., et al. 1998, Nature, 395, 670

Galliano, E., Alloin, D., Pantin, E., et al. 2008, A\&A, 492, 3

Giveon, U., Sternberg, A., Lutz, D., Feuchtgruber, H., \& Pauldrach, A. W. A. 2002, ApJ, 566, 880

Gordon, K. D., Engelbracht, C. W., Fadda, D., et al. 2007, PASP, 119, 1019

Gordon, K. D., Engelbracht, C. W., Rieke, G. H., et al. 2008, ApJ, 682, 336

Gordon, K. D., Rieke, G. H., Engelbracht, C. W., et al. 2005, PASP, 117, 503

Greiner, J., Krühler, T., Fynbo, J. P. U., et al. 2009, ApJ, 693, 1610

Groves, B., Nefs, B., \& Brandl, B. 2008, MNRAS, 391, L113

Gvaramadze, V. V., \& Gualandris, A. 2011, MNRAS, 410, 304

Haan, S., Surace, J. A., Armus, L., et al. 2011, AJ, 141, 100

Hammer, F., Flores, H., Schaerer, D., et al. 2006, A\&A, 454, 103

Han, X. H., Hammer, F., Liang, Y. C., et al. 2010, A\&A, 514, 24

Hao, L., Wu, Y., Charmandaris, V., et al. 2009, ApJ, 704, 1159

Heger, A., Fryer, C. L., Woosley, S. E., Langer, N., \& Hartmann, D. H. 2003, ApJ, 591,288

Higdon, S. J. U., Devost, D., Higdon, J. L., et al. 2004, PASP, 116, 975

Hirschi, R., Meynet, G., \& Maeder, A. 2005, A\&A, 443, 581

Hjorth, J., Thomsen, B., Nielsen, S. R., et al. 2002, ApJ, 576, 113

Holmberg, E. B., Lauberts, A., Schuster, H.-E., \& West, R. M. 1977, A\&AS, 27,295
Houck, J. R., Charmandaris, V., Brandl, B. R., et al. 2004a, ApJS, 154, 211

Houck, J. R., Roellig, T. L., van Cleve, J., et al. 2004b, ApJS, 154, 18

Hunt, L., Bianchi, S., \& Maiolino, R. 2005, A\&A, 434, 849

Hwang, H. S., Elbaz, D., Magdis, G., et al. 2010, MNRAS, 409, 75

Ilbert, O., Arnouts, S., McCracken, H. J., et al. 2006, A\&A, 457, 841

Ivison, R. J., Greve, T. R., Dunlop, J. S., et al. 2007, MNRAS, 380, 199

Kistler, M. D., Yüksel, H., Beacom, J. F., Hopkins, A. M., \& Wyithe, J. S. B. 2009, ApJ, 705, L104

Kocevski, D., West, A. A., \& Modjaz, M. 2009, ApJ, 702, 377

Kouveliotou, C., Meegan, C. A., Fishman, G. J., et al. 1993, ApJ, 413, L101

Lagache, G., Dole, H., Puget, J.-L., et al. 2004, ApJS, 154, 112

Lamb, D. Q., \& Reichart, D. E. 2000, ApJ, 536, 1

Laurent, O., Mirabel, I. F., Charmandaris, V., et al. 2000, A\&A, 359, 887

Le Floc'h, E., Charmandaris, V., Forrest, W. J., et al. 2006, ApJ, 642, 636

Le Floc'h, E., Duc, P.-A., Mirabel, I. F., et al. 2003, A\&A, 400, 499

Lebouteiller, V., Barry, D. J., Spoon, H. W. W., et al. 2011a, ApJS, 196, 8

Lebouteiller, V., Bernard-Salas, J., Whelan, D. G., et al. 2011b, ApJ, 728, 45

Lee, J. H., Hwang, N., \& Lee, M. G. 2011, ApJ, 735, 75

MacFadyen, A. I., \& Woosley, S. E. 1999, ApJ, 524, 262

Marshall, J. A., Herter, T. L., Armus, L., et al. 2007, ApJ, 670, 129

Mathis, J. S. 1990, ARA\&A, 28, 37

Meynet, G., \& Maeder, A. 2005, A\&A, 429, 581

Michałowski, M. J., Hjorth, J., Malesani, D., et al. 2009, ApJ, 693, 347

Modjaz, M., Kewley, L., Kirshner, R. P., et al. 2008, AJ, 135, 1136

Niino, Y., Choi, J.-H., Kobayashi, M. A. R., et al. 2011, ApJ, 726, 88

Podsiadlowski, P., Mazzali, P. A., Nomoto, K., Lazzati, D., \& Cappellaro, E. 2004, ApJ, 607, L17

Rieke, G. H., Young, E. T., Engelbracht, C. W., et al. 2004, ApJS, 154, 25

Rigby, J. R., \& Rieke, G. H. 2004, ApJ, 606, 237

Roussel, H., Sauvage, M., Vigroux, L., \& Bosma, A. 2001, A\&A, 372, 427

Salpeter, E. E. 1955, ApJ, 121, 161

Savaglio, S., Glazebrook, K., \& LeBorgne, D. 2009, ApJ, 691, 182

Schaerer, D., Contini, T., \& Kunth, D. 1999, A\&A, 341, 399

Schaerer, D., \& Stasińska, G. 1999, A\&A, 345, L17

Siebenmorgen, R., \& Krügel, E. 2007, A\&A, 461, 445

Smith, J. D. T., Draine, B. T., Dale, D. A., et al. 2007, ApJ, 656, 770

Sokolov, V. V., Fatkhullin, T. A., Castro-Tirado, A. J., et al. 2001, A\&A, 372 438

Stanek, K. Z., Matheson, T., Garnavich, P. M., et al. 2003, ApJ, 591, L17

Stansberry, J. A., Gordon, K. D., Bhattacharya, B., et al. 2007, PASP, 119, 1038

Stetson, P. B. 1987, PASP, 99, 191

Svensson, K. M., Levan, A. J., Tanvir, N. R., Fruchter, A. S., \& Strolger, L.-G. 2010, MNRAS, 405, 57

Thöne, C. C., Fynbo, J. P. U., Östlin, G., et al. 2008, ApJ, 676, 1151

Totani, T., Kawai, N., Kosugi, G., et al. 2006, PASJ, 58, 485

Vreeswijk, P. M., Fruchter, A., Kaper, L., et al. 2001, ApJ, 546, 672

Ward-Thompson, D., \& Robson, E. I. 1990, MNRAS, 244, 458

Watson, D., French, J., Christensen, L., et al. 2011, ApJ, 741, 58

Werner, M. W., Becklin, E. E., Gatley, I., et al. 1978, MNRAS, 184, 365

Woosley, S. E. 1993, ApJ, 405, 273

Wu, Y., Charmandaris, V., Hao, L., et al. 2006, ApJ, 639, 157 